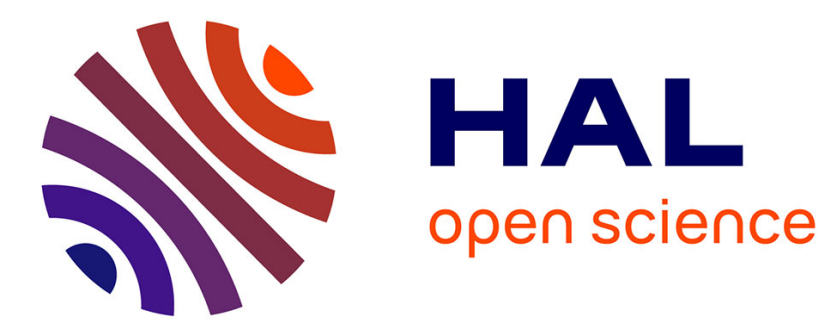

\title{
A Robust Baseband Transceiver Design for Doubly-Dispersive Channels
}

Roberto Bomfin, Marwa Chafii, Ahmad Nimr, Gerhard Fettweis

\section{To cite this version:}

Roberto Bomfin, Marwa Chafii, Ahmad Nimr, Gerhard Fettweis. A Robust Baseband Transceiver Design for Doubly-Dispersive Channels. IEEE Transactions on Wireless Communications, 2021, pp.116. 10.1109/TWC.2021.3062263 . hal-03161973

\section{HAL Id: hal-03161973 https://hal.science/hal-03161973}

Submitted on 8 Mar 2021

HAL is a multi-disciplinary open access archive for the deposit and dissemination of scientific research documents, whether they are published or not. The documents may come from teaching and research institutions in France or abroad, or from public or private research centers.
L'archive ouverte pluridisciplinaire HAL, est destinée au dépôt et à la diffusion de documents scientifiques de niveau recherche, publiés ou non, émanant des établissements d'enseignement et de recherche français ou étrangers, des laboratoires publics ou privés. 


\title{
A Robust Baseband Transceiver Design for Doubly-Dispersive Channels
}

\author{
Roberto Bomfin, Graduate Student Member, IEEE, Marwa Chafii, Member, IEEE, Ahmad Nimr, Member, IEEE, \\ and Gerhard Fettweis, Fellow, IEEE
}

\begin{abstract}
In this paper, we investigate three different concepts for robust link-level performance under doubly-dispersive wireless channels, namely, i) channel estimation, ii) cyclic prefix (CP)free transmission, and iii) waveform design. We employ a unique word-based channel estimation, where we decouple the channel related errors into channel estimation error (CEE) and Doppler error (DE). Then, we show that a trade-off between CEE and DE emerges in the frame design, where the system can be optimized to achieve the minimum composite channel error. Another strategy to improve the link-level performance is to suppress the $\mathbf{C P}$ of the sub-blocks. This allows for better channel estimation due to the reduced transmission time, with the penalty of requiring the CP-restoration processing at the receiver. Furthermore, we propose the waveform design based on the equal-reliability criterion (ERC), leading to the block multiplexing-orthogonal chirp division multiplexing (BM-OCDM). This waveform is advantageous in the CP-free transmission mode, where the data symbols have equally distributed interference from adjacent sub-blocks. Our framework is a generalization of the recently proposed orthogonal time frequency space (OTFS), which fails to achieve the ERC. The link-level simulations show that at high modulation and coding scheme, the proposed BM-OCDM provides superior link-level performance than OTFS.
\end{abstract}

Index Terms-Channel estimation; Iterative receiver; OCDM; OTFS; Doubly-dispersive channel

\section{INTRODUCTION}

Modern mobile communication systems have adopted orthogonal frequency division multiplexing (OFDM) as the main modulation scheme due to its low-complexity implementation for frequency-selective channels. The cyclic prefix (CP) addition in the time domain allows one tap equalization in the frequency domain, which facilitates the receiver implementation. Additionally, OFDM can be employed in multipleinput multiple-output (MIMO) schemes with relatively low complexity. Therefore, OFDM is used in the Long-Term Evolution (LTE) mobile wireless standard and remains the main waveform for the 5th generation (5G) mobile systems [1], [2]. On the other hand, the drawbacks of OFDM are well known and are still investigated by the research community. For instance, one major OFDM issue is its sensitivity to time-varying channels. That is, when the channel changes considerably

Roberto Bomfin, Ahmad Nimr, and Gerhard Fettweis are with the Vodafone Chair Mobile Communication Systems, Technische Universitat Dresden, Germany (e-mail: \{roberto,bomfin, ahmad.nimr, gerhard.fettweis $@ @$ ifn.et.tudresden.de) Marwa Chafii is with ETIS, UMR8051, CY Cergy Paris Université, ENSEA, CNRS, France (e-mail: \{marwa.chafii\}@ensea.fr). This project has received funding from the European Union's Horizon 2020 research and innovation programme through the project iNGENIOUS under grant agreement No 957216, and the CY Initiative of Excellence through the ASIA Chair of Excellence Grant (PIA/ANR-16-IDEX-0008). during an OFDM transmission block, the orthogonality among subcarriers is broken and the system performance is decreased considerably. This phenomenon is significant when the OFDM transmission block exceeds the channel coherence time. In addition, it is known that one-tap equalization allowed by OFDM comes at the cost of reduced performance. Based on that, although OFDM is very appealing complexity-wise, the authors in [3] have shown that single-carrier (SC) waveforms provide higher spectral efficiency than OFDM for frequency-selective channels, using BPSK or QPSK. For higher modulation orders, such as 16-QAM and 32-QAM, numerical simulations also indicate superior performance of SC in terms of information rate. Furthermore, we have shown in [4] that the system performance is optimal, when the data symbols experience the same channel gain, under the assumptions that i) channel state information (CSI) is only available at the receiver, and ii) an iterative equalizer with perfect feedback is used. The latter condition has been demonstrated numerically in [5] for the orthogonal chirp division multiplexing (OCDM) waveform under frequency-selective channels, and in [3] for SC. The spreading concept has recently been used for doubly-dispersive channels. A novel modulation scheme called orthogonal time frequency space (OTFS) has been proposed in [6], where the data symbols are modulated in the delay-Doppler domain [7]. Also, the works [8], [9] have investigated waveforms with equal-gain and iterative equalization. In particular, it has been shown that Walsh-Hadamard (WH) waveform spreads overall time and frequency resources, ultimately achieving the same performance as OTFS. More recently, we have proposed a sparse WH waveform in [10], where we have shown that the equal-gain is achieved with sparse spreading, which can be implemented with lower complexity. In summary, our results in [4], [5], [10] and the analysis of [8], [9] are complementary approaches that lead to the same conclusion: data symbols should experience the same channel gain to maximize performance. In this paper, we denote this condition as equal gain criterion (EGC).

Another important aspect for a realistic analysis of transmission under doubly-dispersive channels is channel estimation. In this paper, we consider a unique word (UW) based channel estimation scheme [11], [12], which is easily extended to MIMO and has smaller overhead than pilot-based channel estimation. Originally, the idea of the UW channel estimation refers to replacing the $\mathrm{CP}$ in the data block by a known sequence, where the UW is transmitted before and after the data block. However, we also consider the case where the CP can be included in the data block. This can be interpreted 
as a generalization of the pilot block channel estimation of [13], because our UW has flexible length. In this paper, we show that the system is impaired by two channel related errors, namely, channel estimation error and Doppler spread. A tradeoff between this two errors is established, where a frame optimization tool is proposed to minimize the overall channel related errors.

Lastly, in [14] we have proposed an iterative receiver for $\mathrm{CP}$ free transmission under frequency-selective channels, which can be applied to systems under doubly-dispersive channels. Basically, the CP-free system reduces the overall transmission time when the frame is composed of multiple sub-blocks, which consequently improves the UW or pilot block based channel estimation. The drawback of this technique is the additional interference introduced among the sub-blocks, which is tackled by the CP-Restoration procedure. In [12], we have also considered CP-free transmission with full CP-Restoration of [15]. However, we have demonstrated that this solution provides inferior performance than the scheme of [14]. In this paper, we show that the $\mathrm{CP}$-free transmission has superior performance than conventional $\mathrm{CP}$ systems.

Lastly, based on the EGC for the system with $\mathrm{CP}$, we provide a waveform design for the $\mathrm{CP}$-free condition. In this case, the data symbols experience the same channel gain and same interference level. This is called equal reliability condition (ERC), as it is a generalization of EGC when the data symbols suffer from localized interference. We show that the ERC is achieved by the block multiplexing (BM)-OCDM waveform.

In this paper, we consider the aforementioned ideas under the same framework in order to provide a robust transmission scheme for doubly-dispersive channels. Accordingly, the contributions are listed in the following.

- We provide a theoretical tool to optimize the transmitted frame. We extend the work of [13] by considering the Doppler error caused by the time varying channel within one data block, while [13] assumes that the channel is static during one data block transmission. Thus, we establish a trade-off between CEE and DE. We provide the theoretical expressions for these errors which are useful for two reasons: i) we can minimize the composite channel error by setting the correct frame structure, i.e., the amount of sub-blocks utilized for data transmission, and ii) the knowledge of these errors is required by the equalizer.

- We extend the idea of our CP-free concept in [14] by considering realistic channel estimation. We demonstrate that in highly time-variant channels, it is advantageous to not include CPs in the data sub-blocks, yielding to improved channel estimation and hence, improved link level performance.

- We consider waveform design in conjunction with the CP-free transmission. The findings of [4]-[6], [13] show that spreading symbols over time and frequency provides robust link level performance due to equal channel gains experienced by the symbols. However, if we transmit the data without $\mathrm{CP}$, there is interference from the adjacent sub-blocks. Therefore, in addition to the equal-gain, we should also aim for this interference to be equally distributed among the data symbols. This principle, referred as ERC, is achieved by multiplexing OCDM modulation to achieve perfect time and frequency spreading.

The rest of this paper is organized as follows. In Section II, we provide a high-level description of the system model. In Section III, we describe the channel estimation approach and define frame optimization problem. Section IV presents the waveform design for CP-free transmission based on the ERC. Section V defines the LMMSE-PIC iterative equalizer and its low-complexity implementation. In Section VI, we present our numerical evaluations, and we conclude the paper in Section VII.

Notations: Unless otherwise stated, vectors are defined with lowercase bold symbols $\mathbf{x}$ whose $n$-th element is $(\mathbf{x})_{n}$. Matrices are written as uppercase bold symbols $\mathbf{X}$ whose element in $n$-th row and $n^{\prime}$-th column is $(\mathbf{X})_{n, n^{\prime}}$. If $\mathbf{x}_{0}$ and $\mathbf{x}_{1}$ are two vectors of same size, the product $\mathbf{x}_{0} \circ \mathbf{x}_{1}$ and the division $\mathbf{x}_{0} / \mathbf{x}_{1}$ are performed element-wise returning a vector of the same size as $\mathbf{x}_{0}$ and $\mathbf{x}_{1}$. We consider the special matrices, i) $\mathbf{F}_{K}$ is the normalized Fourier matrix of size $K$, ii) $\mathbf{I}_{K}$ is the identity matrix of size $K$, iii) $\mathbf{0}_{N_{0}, N_{1}}$ is a zero matrix of size $N_{0} \times N_{1}$, iv) $\mathbf{1}_{N}$ is a column vector of ones with size $N$. We consider the special functions, i) $\operatorname{Tr}(\mathbf{X})$ is the trace of $\mathbf{X}$, ii) $\operatorname{diag}(\mathbf{X})$ returns a column vector whose elements are the diagonal of $\mathbf{X}$, iii) $\delta[n]=1$ for $n=0$ and zero otherwise, iv) shift $_{N_{0}, N_{1}}\{\mathbf{X}\}$ performs a circular shift over the rows and columns of $\mathbf{X}$ by and amount of $N_{0}$ and $N_{1}$, respectively. $\mathbf{X}^{\mathrm{T}}$ and $\mathbf{X}^{\mathrm{H}}$ returns the transpose and complex conjugate of $\mathbf{X}$, respectively. Finally, $\mathbb{E}\{\cdot\}$ stands for the expectation operator.

\section{SySTEM MODEL}

\section{A. Transmitter}

Consider a bit-interleaved coded modulation system, where a vector of information bits $\mathbf{b} \in\{0,1\}^{N_{\mathrm{b}}}$ is encoded generating the coded bit stream $\mathbf{c} \in\{0,1\}^{N_{\mathrm{c}}}$, being $N_{\mathrm{b}}$ and $N_{\text {c }}$ the amount of uncoded and coded bits, respectively. In the sequence, the coded bits $\mathbf{c}$ are interleaved and mapped onto a quadrature amplitude modulation (QAM) constellation set $\mathcal{S}$ with QAM order $|\mathcal{S}|=J$. As such, the data vector $\mathbf{d} \in \mathcal{S}^{N}$ is generated, where $N=N_{\mathrm{c}} /\left(\log _{2} J\right)$ is the amount of symbols per transmission with $\mathbb{E}\left\{\mathbf{d d}^{\mathrm{H}}\right\}=\mathbf{I}_{N}$. The data vector is linearly modulated as $\mathbf{x}=\mathbf{A d}$, which can be reformulated as a concatenation of $M$ sub-blocks with size $K$ as $\mathbf{x}=\left(\mathbf{x}_{0}^{\mathrm{T}}, \mathbf{x}_{1}^{\mathrm{T}}, \cdots, \mathbf{x}_{M-1}^{\mathrm{T}}\right)^{\mathrm{T}}$, for $\mathbf{x}_{m}=[\mathbf{x}]_{n=m K+1}^{(m+1) K} \in \mathbb{C}^{K}$, such that $N=K M$. Moreover, $\mathbf{A} \in \mathbb{C}^{N \times N}$ is regarded as the linear modulation matrix. A detailed structure of $\mathbf{A}$ and its implications in the $\mathrm{CP}$ and $\mathrm{CP}$-free transmission under doubly dispersive channel are discussed in Section IV.

In this paper, a UW-based channel estimation approach [16], [17] is considered, where the deterministic UW signal is always transmitted before and after the data blocks. In addition, we consider two different transmission modes, namely, with and without the addition of a CP before each sub-block $\mathbf{x}_{m}$. Throughout this paper, we will denote the transmission without $\mathrm{CP}$ as CP-free. For transmission with $\mathrm{CP}$, the sub-blocks $\mathbf{x}_{m}$ are modified as $\tilde{\mathbf{x}}_{m}=\left(\left[\mathbf{x}_{m}^{\mathrm{T}}\right]_{n=N-N_{\mathrm{CP}}+1}^{N}, \mathbf{x}_{m}^{\mathrm{T}}\right)^{\mathrm{T}}$, where $N_{\mathrm{CP}}$ 


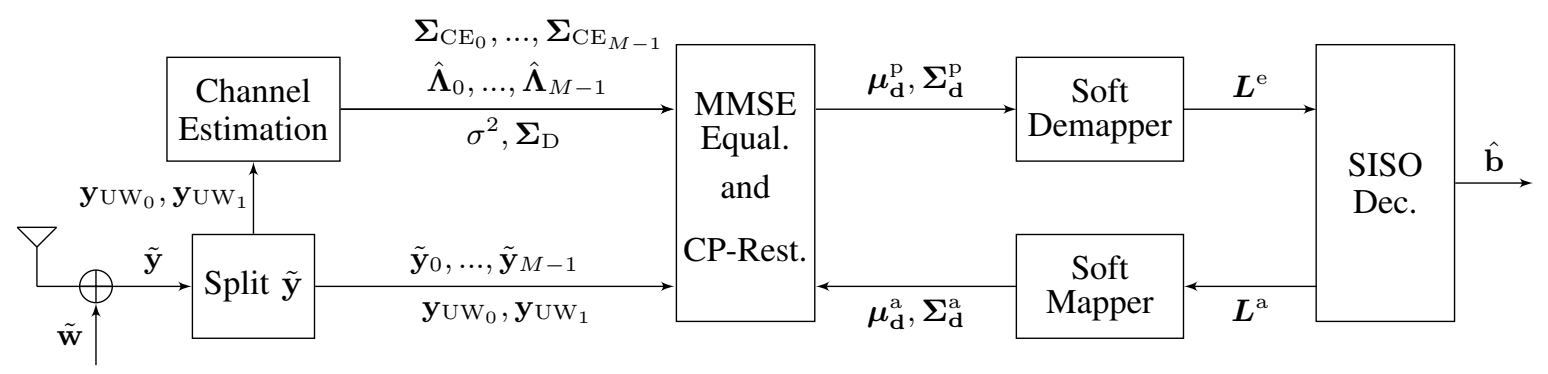

Fig. 1. Block diagram of the receiver.

is greater than the number of the channel taps. The CP-free system is obtained by making $N_{\mathrm{CP}}=0$. The transmitted signal including the UW for the two transmission modes is determined as

$$
\mathbf{x}_{\text {frame }}=\left(\mathbf{x}_{\mathrm{UW}}^{\mathrm{T}}, \tilde{\mathbf{x}}_{0}^{\mathrm{T}}, \cdots, \tilde{\mathbf{x}}_{M-1}^{\mathrm{T}}, \mathbf{x}_{\mathrm{UW}}^{\mathrm{T}}\right)^{\mathrm{T}},
$$

where the UW signal is defined by the Zadoff-Chu (ZC) sequence $\mathbf{x}_{\mathrm{UW}}[n]=\exp \left(j \pi n^{2} / N_{\mathrm{UW}}\right)$, for $n=-N_{\mathrm{CP}_{\text {uw }}}, \cdots, N_{\mathrm{UW}}-1$. This signal is commonly used for channel estimation [13], [17]. Note that $\mathbf{x}_{U W}$ has a length of $N_{\mathrm{CP}_{\text {uw }}}+N_{\mathrm{UW}}$ regardless of the transmission mode, and it is different from the size of $\mathbf{x}_{m}$ in general. If $N_{\mathrm{UW}}=N_{\mathrm{CP}_{\mathrm{uw}}}$, the UWs become equivalent to the sequences employed in [16].

\section{B. Doubly-Dispersive Wireless Channels}

In this work, we consider a doubly selective wireless channel whose impulse response at the $n$-th time index is

$$
\mathbf{h}_{n}=\left(h_{n, 0}, h_{n, 1}, \cdots, h_{n, L-1}\right)^{\mathrm{T}},
$$

where $h_{n, l}$ is a complex Gaussian process stationary with respect to (w.r.t.) $n$ and uncorrelated w.r.t. $l$, with a Rayleighdistributed envelope and uniformly distributed phase between 0 and $2 \pi$. The variable $L$ denotes the channel length. We model the $l$-th path as a random process with the correlation function given by

$$
\mathbb{E}\left\{h_{n, l} h_{n^{\prime}, l^{\prime}}^{\mathrm{H}}\right\}=\left\{\begin{array}{cc}
\rho_{l} \Upsilon\left(n-n^{\prime}\right) & , l=l^{\prime} \\
0 & , l \neq l^{\prime}
\end{array}\right.
$$

which is based on the well known Clarke's model [18] with following correlation

$$
\Upsilon\left(\Delta_{n}\right)=J_{0}\left(\frac{2 \pi \Delta_{n} f_{\mathrm{d}}}{B}\right),
$$

where $J_{0}(\cdot)$ is the zero-th order Bessel function of the first kind, $B$ is the bandwidth, $f_{\mathrm{d}}=f_{\mathrm{c}} v / c$ is the maximum Doppler spread which depends on the speed difference $v$ between transmitter and receiver, the speed of light $c$ and the carrier frequency $f_{\mathrm{c}}$. Also, we implicitly assume no correlation among the channel taps and $\rho_{l}$ model the power of the $l$-th path according to a given power delay profile.

\section{Receiver Structure}

The receiver structure is depicted in Figure 1. The signals generated by the Split block are defined later on in this section. The Channel Estimation block is explained in Section III. The block MMSE Equal. and CP-Rest. is described in V. The blocks Soft Demapper and Soft Mapper are taken from [5]. Finally, the SISO Dec. block is based on the Log-MAP BCJR SISO Decoder.

Under the simplifying assumption of perfect synchronization, the discrete-time received signal is given by

$$
\tilde{\mathbf{y}}[n]=\sum_{l=0}^{L-1} \mathbf{h}_{n}[l] \mathbf{x}_{\text {frame }}[n-l]+\tilde{\mathbf{w}}[n],
$$

where $\mathbf{w} \sim \mathcal{C N}\left(0, \sigma^{2} \mathbf{I}\right)$ is the additive white Gaussian noise with variance $\sigma^{2}$. The receiver splits $\tilde{\mathbf{y}}$ into two parts, namely, the respective UW signals and the data signals. The former is forwarded to the channel estimation block, which is discussed in Section III. The latter is sent to the MMSE equalizer block, which is discussed in Section V. Then, the first and last UWs are respectively extracted from $\tilde{\mathbf{y}}$ as follows

$$
\mathbf{y}_{\mathrm{UW}_{0}}=[\tilde{\mathbf{y}}]_{n=N_{\mathrm{CP} \text { uw }}}^{N_{\mathrm{CP}_{\mathrm{uw}}}+N_{\mathrm{UW}}-1}=\mathbf{H}_{\mathrm{UW}_{0}} \mathbf{x}_{\mathrm{UW}}+\mathbf{w}_{\mathrm{UW}_{0}},
$$

and

$$
\mathbf{y}_{\mathrm{UW}_{1}}=[\tilde{\mathbf{y}}]_{n=N_{\mathrm{CP}} N_{\mathrm{uw}}+\Delta_{\mathrm{UW}}}^{N_{\mathrm{CP}}+\Delta_{\mathrm{UW}}}=\mathbf{H}_{\mathrm{UW}_{1}} \mathbf{x}_{\mathrm{UW}}+\mathbf{w}_{\mathrm{UW}_{1}},
$$

where $\Delta_{\mathrm{UW}}=N_{\mathrm{CP}_{\mathrm{UW}}}+N_{\mathrm{UW}}+M\left(K+N_{\mathrm{CP}}\right)$ is the amount of samples between the consecutive UWs. The vectors $\mathbf{w}_{U W_{0}}$ and $\mathbf{w}_{\mathrm{UW}_{1}}$ correspond to the respective AWGN terms of the first and last UWs. The channel matrices operating on the UWs are filled with the channel coefficients as

$$
\mathbf{H}_{\mathrm{UW}_{u}}=\tilde{\mathbf{H}}\left(N_{\mathrm{CP}_{\mathrm{UW}}}+u \Delta_{\mathrm{UW}}, N_{\mathrm{UW}}\right),
$$

where the matrix $\tilde{\mathbf{H}}\left(n_{\text {ini }}, N_{\text {UW }}\right) \in \mathbb{C}^{N_{\text {UW }} \times N_{\text {Uw }}}$ is defined in (9) in the bottom of next page, and is a quasi-circulant ${ }^{1}$ channel matrix due to $\mathrm{CP}$ insertion. The quantity $n_{\text {ini }}$ represents the initial channel sample and $T=N_{\mathrm{UW}}$ in (9) is due to the UW length. Additionally, we denote $u=0$ for the first UW, and $u=1$ for the last $\mathrm{UW}$.

\footnotetext{
${ }^{1}$ By quasi-circulant, we mean that the channel has similar structure as a circulant matrix due to the CP-insertion. However, due to the time-varying nature of the channel's impulse response, $\tilde{\mathbf{H}}\left(n_{\text {ini }}, T\right)$ is not a perfect circulant matrix in general.
} 
Similarly, the samples related to the $m$-th sub-block are extracted from $\tilde{\mathbf{y}}$ as

$$
\mathbf{y}_{m}=[\tilde{\mathbf{y}}]_{n=N_{\mathrm{ini}_{m}}}^{N_{\mathrm{ini}_{m}}+K},
$$

where the initial sample of the received $m$-th block is defined as $N_{\mathrm{ini}_{m}}=N_{\mathrm{CP}_{\text {uw }}}+N_{\mathrm{UW}}+N_{\mathrm{CP}}+m\left(N_{\mathrm{CP}}+K\right)$. In the following, we define the received signal for the $\mathrm{CP}$ and $\mathrm{CP}$ free transmission.

1) $C P$ : For the transmission with $\mathrm{CP}$, the received signal can be formulated as

$$
\tilde{\mathbf{y}}_{\mathrm{CP}_{m}}=\mathbf{H}_{m} \mathbf{x}_{m}+\mathbf{w}_{m},
$$

where $\mathbf{H}_{m}=\tilde{\mathbf{H}}\left(N_{\text {ini }_{m}}, K\right) \in \mathbb{C}^{K \times K}$ and the CP removal at the receiver side is performed.

2) $C P$-free: For CP-free transmission, the channel matrix can be written as

$$
(\tilde{\mathbf{H}})_{n, n^{\prime}}=\left\{\begin{array}{ll}
\left(\mathbf{H}_{m}\right)_{n, n^{\prime}}, & n \geq n^{\prime} \\
0, & n<n^{\prime}
\end{array},\right.
$$

with zeros in the upper triangular components. Then, the received samples for the $m$-th sub-block are [14]

$$
\tilde{\mathbf{y}}_{m}=\tilde{\mathbf{H}}_{m} \mathbf{x}_{m}+\left(\mathbf{H}_{m-1}-\tilde{\mathbf{H}}_{m-1}\right) \mathbf{x}_{m-1}+\mathbf{w}_{m},
$$

where the component $\left(\mathbf{H}_{m-1}-\tilde{\mathbf{H}}_{m-1}\right) \mathbf{x}_{m-1}$ represents the interference from the $(m-1)$ th sub-block.

\section{Channel Estimation And Frame Optimization}

The channel estimation for doubly dispersive channels considered in this paper is an extension of the one provided in [13] in two ways. Firstly, we consider a reduced size pair of UWs as our pilot blocks, while [13] considers an entire pilot block for channel estimation, which can be energy inefficient and can produce higher errors because the estimated channel per UW is more susceptible to inter carrier interference (ICI) cause by the Doppler spread, which is mitigated by reducing the UW size. Secondly, our model considers that the channel is also varying within one data block, whereas [13] assumes that the channel remains static for the duration of each data block. This second extension is decisive for deriving a framework for the design of a more robust frame structure in highly timeselective channels.

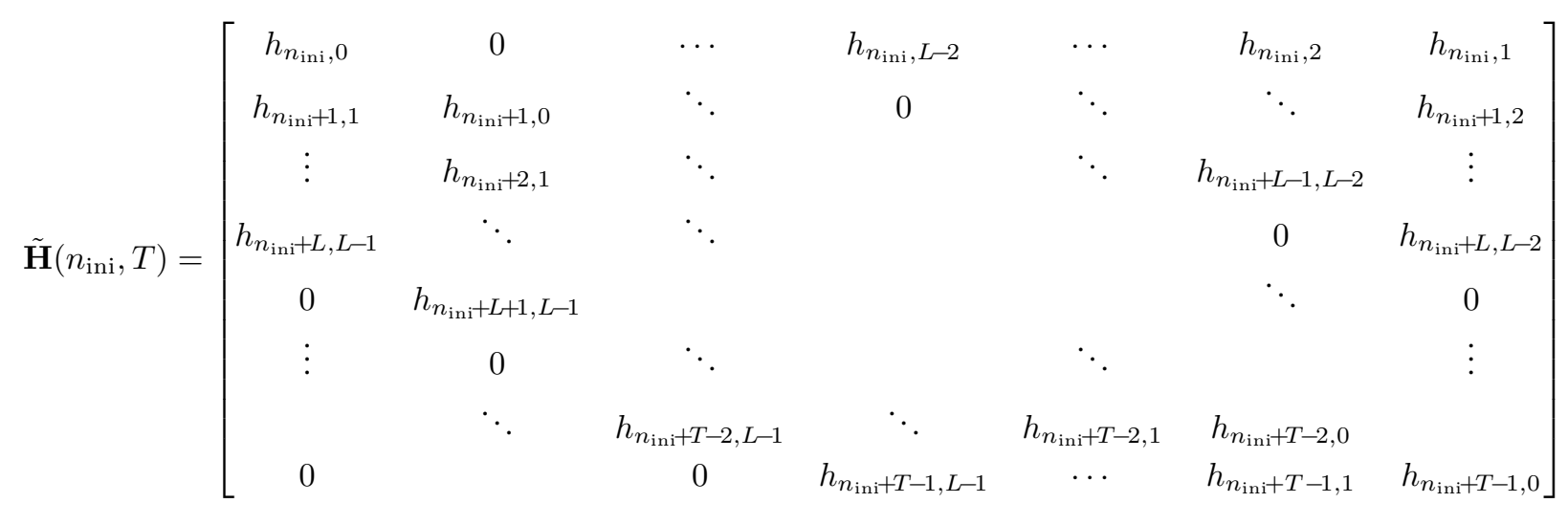

\section{A. LS-based Frequency Domain Channel Estimation}

Since the equalizers used in this paper perform the equalization in the frequency domain (FD), we consider FD channel estimation. In short, we estimate the frequency response of the channel for both UWs using the least-square method, similarly to [13]. Then, the frequency components of these estimates are interpolated in order to provide a channel estimation for the data blocks. Initially, the UWs' channels in FD $\hat{\Lambda}_{\mathrm{UW}_{u}}^{\prime} \in \mathbb{C}^{N_{\mathrm{UW}}}$ are estimated as the element-wise division

$$
\hat{\Lambda}_{\mathrm{UW}_{u}}^{\prime}=\frac{\mathbf{Y}_{\mathrm{UW}_{u}}}{\mathbf{X}_{\mathrm{UW}}},
$$

where $u=0$ and $u=1$ denote the first and last UWs, respectively. $\mathbf{Y}_{\mathrm{UW}_{u}} \in \mathbb{C}^{N_{\mathrm{UW}}}=\mathbf{F}_{N_{\mathrm{UW}}} \mathbf{y}_{\mathrm{UW}_{u}}$ is the received UW signals defined in (6) and (7) transformed to the FD. Similarly, $\mathbf{X}_{\mathrm{UW}}=\mathbf{F}_{N_{\mathrm{UW}}} \mathbf{x}_{\mathrm{UW}} \in \mathbb{C}^{N_{\mathrm{UW}}}$ transmitted UW signal in FD.

Subsequently, we perform two operations on $\hat{\Lambda}_{\mathrm{UW}}^{\prime}$. Initially, we filter this estimate in time domain, such that the channel samples beyond $L$ are nullified and the mean squared error is reduced [13]. After that, we set the size of the resulting matrix to match the same length $K$ as the data sub-blocks, such that we can use these estimates in the FD equalization. These two operations are mathematically described as

$$
\hat{\boldsymbol{\Lambda}}_{\mathrm{UW}_{u}}=\mathbf{F}_{K}\left(\mathbf{D}\left(\mathbf{F}_{N_{\mathrm{UW}}}^{\mathrm{H}} \hat{\boldsymbol{\Lambda}}_{\mathrm{UW}_{u}}^{\prime}\right)\right),
$$

resulting in $\hat{\boldsymbol{\Lambda}}_{\mathrm{UW}_{u}} \in \mathbb{C}^{K}$. We first perform an $N_{\mathrm{UW}}$-sized IFFT in the inner parenthesis. Then we filter the resulting time domain channel estimate with the matrix $\mathbf{D}$

$$
\mathbf{D}=\left[\begin{array}{cc}
\mathbf{I}_{L} & \mathbf{0}_{L, L-N_{\mathrm{UW}}} \\
\mathbf{0}_{N-L, L} & \mathbf{0}_{N-L, L-N_{\mathrm{UW}}}
\end{array}\right],
$$

which achieves the two aforementioned required operations: i) time filtering with an $L$-sized window, and ii) appending zeros to the time domain estimate to force a column vector of size $K$. Finally, a $K$-sized FFT is performed in (8) to bring the estimate to FD. As in [13], we estimate the $k$-th frequency point of the $m$-th data block as a linear combination of the estimated channel for both $\mathrm{UWs}^{2}$

$$
\left(\hat{\mathbf{\Lambda}}_{m}\right)_{k, k}=\mathbf{C}_{m, k} \hat{\mathbf{P}}_{k}
$$

\footnotetext{
${ }^{2}$ Note that for notational simplicity, we define the UW related channels $\hat{\boldsymbol{\Lambda}}_{\mathrm{UW}_{u}}^{\prime}$ and $\hat{\boldsymbol{\Lambda}}_{\mathrm{UW}_{u}}$ as vector quantities. Hereafter, we consider the channel estimates in FD as matrices.
} 
for all $m=0, \cdots, M-1$ and $k=0, \cdots K-1$, where the off-diagonal elements of $\hat{\boldsymbol{\Lambda}}_{m}$ are zero in order to allow a simple one tap equalization in FD. Also, the column vector $\hat{\mathbf{P}}_{k}=\left(\left(\hat{\boldsymbol{\Lambda}}_{\mathrm{UW}_{0}}\right)_{k},\left(\hat{\boldsymbol{\Lambda}}_{\mathrm{UW}_{1}}\right)_{k}\right)^{\mathrm{T}}$ contains the estimated channel of both UWs for the $k$-th frequency component, and the row vector $\mathbf{C}_{m, k}=\left(C_{m, k}^{0}, C_{m, k}^{1}\right)$ contains the linear interpolation coefficients, which are computed such that the error $\sigma_{\mathrm{CE}_{m, k}}^{2}=$ $\mathbb{E}\left\{\left|\left(\hat{\boldsymbol{\Lambda}}_{m}\right)_{k, k}-\left(\boldsymbol{\Lambda}_{m}\right)_{k, k}\right|^{2}\right\}$ is minimized. The closed form solution for $\mathbf{C}_{m, k}$ is derived in the Appendix $\mathrm{A}$ and is given in equation (18). Finally, we highlight that this channel estimation scheme allows a straightforward generalization to MIMO similarly to [12], where shifted versions of the ZC sequence can be used for different antennas. However, this is out of the scope of this paper and is left for future work.

\section{B. Channel Errors}

In order to provide a systematic analysis on the channel errors, we decouple the channel into two parts as

$$
\begin{aligned}
\boldsymbol{\Lambda}_{m} & =\mathbf{F}_{K} \mathbf{H}_{m} \mathbf{F}_{K}^{\mathrm{H}} \\
& =\mathbf{F}_{K} \overline{\mathbf{H}}_{m} \mathbf{F}_{K}^{\mathrm{H}}+\mathbf{F}_{K}\left(\mathbf{H}_{m}-\overline{\mathbf{H}}_{m}\right) \mathbf{F}_{K}^{\mathrm{H}} \\
& =\overline{\boldsymbol{\Lambda}}_{m}+\tilde{\boldsymbol{\Lambda}}_{m},
\end{aligned}
$$

where $\mathbf{H}_{m} \in \mathbb{C}^{K \times K}$ is the quasi-circulant channel matrix of the $m$-th sub-data block defined in (9), and $\overline{\mathbf{H}}_{m} \in \mathbb{C}^{K \times K}$ is a perfect circulant matrix whose first column is the average time-varying impulse response of $\mathbf{H}_{m}$

$$
\left(\overline{\mathbf{H}}_{m}\right)_{n, 0}=\frac{1}{K} \sum_{i=0}^{K-1}\left(\mathbf{H}_{m}\right)_{(i+n)_{K}, i} .
$$

for $n=0, \cdots, K-1$. As such, the term $\overline{\boldsymbol{\Lambda}}_{m}=\mathbf{F}_{K} \overline{\mathbf{H}}_{m} \mathbf{F}_{K}^{\mathrm{H}}$ is diagonal and can be regarded as the static part of the channel. On the other hand, $\tilde{\Lambda}_{m}=\mathbf{F}_{K}\left(\mathbf{H}_{m}-\overline{\mathbf{H}}_{m}\right) \mathbf{F}_{K}^{\mathrm{H}}$ is off-diagonal ${ }^{3}$, and can be interpreted as the time-variant component of the channel, i.e., the ICI matrix caused by the Doppler spread. Next, we show that the decomposition of (19) is fundamental to understand the composite error caused by channel estimation and Doppler spread.

The channel estimation in (17) can be expressed as $\hat{\boldsymbol{\Lambda}}_{m}=$ $\boldsymbol{\Lambda}_{m}+\boldsymbol{\Lambda}_{\mathrm{e}_{m}}$. In combination with (19), the channel error matrix for the $m$-th sub-block $\boldsymbol{\Lambda}_{\mathrm{e}_{m}} \in \mathbb{C}^{K \times K}$ can be decomposed as

$$
\boldsymbol{\Lambda}_{\mathrm{e}_{m}}=\hat{\boldsymbol{\Lambda}}_{m}-\left(\overline{\boldsymbol{\Lambda}}_{m}+\tilde{\boldsymbol{\Lambda}}_{m}\right)=\boldsymbol{\Lambda}_{\mathrm{CEE}_{m}}+\boldsymbol{\Lambda}_{\mathrm{DE}_{m}},
$$

in which $\bar{\Lambda}_{m}+\tilde{\Lambda}_{m}=\Lambda_{m}$ is the channel matrix in FD (19). $\boldsymbol{\Lambda}_{\mathrm{CEE}_{m}}=\left(\hat{\boldsymbol{\Lambda}}_{m}-\overline{\boldsymbol{\Lambda}}_{m}\right)$ is the channel estimation error (CEE)

\footnotetext{
${ }^{3}$ We omit the proof due to lack of space. Based on the construction of $\mathbf{F}_{K}$, one can show that $\operatorname{diag}\left(\mathbf{F}_{K} \mathbf{H}_{m} \mathbf{F}_{K}^{\mathrm{H}}\right)=\operatorname{diag}\left(\mathbf{F}_{K} \overline{\mathbf{H}}_{m} \mathbf{F}_{K}^{\mathrm{H}}\right)$, which makes $\tilde{\Lambda}_{m}$ to be off-diagonal.
}

matrix. Obviously, $\boldsymbol{\Lambda}_{\mathrm{CEE}_{m}}$ is diagonal because the channel estimation matrix in (17) is also diagonal. Also, $\boldsymbol{\Lambda}_{\mathrm{DE}_{m}}=$ $-\tilde{\Lambda}_{m}$ is the Doppler error (DE) matrix and is off-diagonal due to (19).

The power related to the channel estimation error $\sigma_{\mathrm{CE}_{m, k}}^{2}=$ $\mathbb{E}\left\{\left(\boldsymbol{\Lambda}_{\mathrm{CEE}_{m}} \boldsymbol{\Lambda}_{\mathrm{CEE}_{m}}^{\mathrm{H}}\right)_{k, k}\right\}$ for the $m$-th sub-block and $k$-th subcarrier is

$$
\begin{aligned}
& \sigma_{\mathrm{CE}_{m, k}}^{2}=\mathbb{E}\left\{\left|\left(\boldsymbol{\Lambda}_{m}\right)_{k, k}\right|^{2}\right\} \\
& -\mathbb{E}\left\{\left(\boldsymbol{\Lambda}_{m}\right)_{k, k} \hat{\mathbf{P}}_{k}^{\mathrm{H}}\right\} \mathbb{E}\left\{\hat{\mathbf{P}}_{k} \hat{\mathbf{P}}_{k}^{\mathrm{H}}\right\}^{-1} \mathbb{E}\left\{\left(\boldsymbol{\Lambda}_{m}\right)_{k, k} \hat{\mathbf{P}}_{k}^{\mathrm{H}}\right\}^{\mathrm{H}},
\end{aligned}
$$

where $\mathbb{E}\left\{\left|\left(\boldsymbol{\Lambda}_{m}\right)_{k, k}\right|^{2}\right\}=1 / K^{2} \sum_{i=0}^{K-1} \sum_{j=0}^{K-1} \Upsilon(i-j) \forall_{k, m}$ is demonstrated in Appendix B. The expectations in the second line of (22) can be directly extracted from equation (18).

The power related to the Doppler spread is $\sigma_{\mathrm{D}_{m, k}}^{2}=$ $\mathbb{E}\left\{\left(\boldsymbol{\Lambda}_{\mathrm{DE}_{m}} \boldsymbol{\Lambda}_{\mathrm{DE}_{m}}^{\mathrm{H}}\right)_{k, k}\right\}$, and can be calculated according to Appendix $\mathrm{C}$ as

$$
\sigma_{\mathrm{D}_{m, k}}^{2}=1-\frac{1}{K^{2}} \sum_{i=0}^{K-1} \sum_{i^{\prime}=0}^{K-1} \Upsilon\left(i-i^{\prime}\right) .
$$

\section{Frame Optimization}

According to the previous derivations, we observe that the data signals are impaired by two different sources of errors, namely, the CEE $\sigma_{\mathrm{CE}_{m, k}}^{2}$ and the $\mathrm{DE} \sigma_{\mathrm{D}_{m, k}}^{2}$. Understanding these two errors is very relevant, because they establish a trade-off between the parameters $K$ and $M$ for the same $N=K M$. For instance, by decreasing $K$, we can expect $\sigma_{\mathrm{D}_{m, k}}^{2}$ to decrease because the time duration of each subblock will decrease, therefore the channel will be more static during the transmission in a sub-block. However, when the transmitter includes the CPs, it will increase the time distance between the two UWs. Therefore, we can expect $\sigma_{\mathrm{CE}_{m, k}}^{2}$ to increase. By defining the average $\mathrm{CEE}$ and $\mathrm{DE}$ respectively as $\sigma_{\mathrm{CE}_{\mathrm{CP}}}^{2}(M)=1 /(M \cdot K) \sum_{m=0}^{M-1} \sum_{k=0}^{K-1} \sigma_{\mathrm{CE}_{m, k}}^{2}$ and $\sigma_{\mathrm{D}}^{2}(M)=1 /(M \cdot K) \sum_{m=0}^{M-1} \sum_{k=0}^{K-1} \sigma_{\mathrm{D}_{m, k}}^{2}$, we can minimize the compound error by choosing the appropriate pair $\{K, M\}$. Therefore, the frame defined in (1) can be optimized by resolving the minimization

$$
\begin{aligned}
\min _{M} & \sigma_{\mathrm{CE}_{\mathrm{CP}}}^{2}(M)+\sigma_{\mathrm{D}}^{2}(M) \\
\text { subject to } & K M=N
\end{aligned}
$$

where $K M=N$ is a fixed quantity and reflects the model presented in Section II, in which the UW is sent before and after the data signal of length $N$. In Section VI, we resolve the minimization in (24) numerically. It is worth noticing that the frame optimization for the $\mathrm{CP}$ systems presented in this subsection is applicable regardless of the waveform because

$$
\begin{aligned}
\mathbf{C}_{m, k}= & {\left[\begin{array}{ll}
\left(\mathbf{F}_{K} \mathbf{D} \mathbf{U}^{\mathrm{H}} \mathbf{S}(m, 0) \mathbf{U} \mathbf{D}^{\mathrm{H}} \mathbf{F}_{K}^{\mathrm{H}}\right)_{k, k} & \left(\mathbf{F}_{K} \mathbf{D} \mathbf{U}^{\mathrm{H}} \mathbf{S}(m, 1) \mathbf{U} \mathbf{D}^{\mathrm{H}} \mathbf{F}_{K}^{\mathrm{H}}\right)_{k, k}
\end{array}\right]^{\mathrm{T}} } \\
& \times\left[\begin{array}{cc}
\left(\mathbf{F}_{K} \mathbf{D} \mathbf{U}^{\mathrm{H}} \mathbf{R}(0,0) \mathbf{U} \mathbf{D}^{\mathrm{H}} \mathbf{F}_{K}^{\mathrm{H}}\right)_{k, k}+\frac{L}{N} \sigma^{2} & \left(\mathbf{F}_{K} \mathbf{D} \mathbf{U}^{\mathrm{H}} \mathbf{R}(0,1) \mathbf{U} \mathbf{D}^{\mathrm{H}} \mathbf{F}_{K}^{\mathrm{H}}\right)_{k, k} \\
\left(\mathbf{F}_{K} \mathbf{D} \mathbf{U}^{\mathrm{H}} \mathbf{R}(1,0) \mathbf{U} \mathbf{D}^{\mathrm{H}} \mathbf{F}_{K}^{\mathrm{H}}\right)_{k, k} & \left(\mathbf{F}_{K} \mathbf{D} \mathbf{U}^{\mathrm{H}} \mathbf{R}(1,1) \mathbf{U} \mathbf{D}^{\mathrm{H}} \mathbf{F}_{K}^{\mathrm{H}}\right)_{k, k}+\frac{L}{N} \sigma^{2}
\end{array}\right]^{-1}
\end{aligned}
$$


$\sigma_{\mathrm{CE}}^{2}(M)+\sigma_{\mathrm{DP}}^{2}(M)$ is independent of the modulation matrix. Lastly, we highlight that optimizing the frame for the CP-free system cannot be achieved by the approach of (24) because the system is impaired with interference among sub-blocks. In this case, besides setting $K$ and $M$, the performance depends on the capability of the receiver to remove this interference.

\section{WAVEFORM DESIGN FOR CP-FREE SYSTEM}

In [14], we have suggested the employment of a CP-free system under doubly dispersive channel, such that the channel estimation is improved. Therefore, in this section we propose a waveform design for the $\mathrm{CP}$-free system, where the data symbols experience the same channel gain and interference level.

\section{A. Block Multiplexing (BM) Structure}

In this paper, the modulation matrix of Section II is defined by the BM structure as

$$
\mathbf{A}=\mathbf{B} \otimes \mathbf{A}^{\prime}
$$

where $\mathbf{A}^{\prime} \in \mathbb{C}^{K \times K}$ is regarded as the modulation matrix per sub-block. In this paper, we name $\mathbf{B} \in \mathbb{C}^{M \times M}$ as the block multiplexing (BM) matrix. We consider that $\mathbf{A}^{\prime}$ and $\mathbf{B}$ are unitary matrices, which also makes $\mathbf{A}$ unitary. In addition, by splitting the data vector $\mathbf{d}^{\mathrm{T}}$ into $\left(\mathbf{d}_{0}^{\mathrm{T}} \cdots \mathbf{d}_{M-1}^{\mathrm{T}}\right)$ with $\mathbf{d}_{m} \in$ $\mathcal{S}^{K}$, we can write the $m$-th modulated sub-block as

$$
\mathbf{x}_{m}=\sum_{m^{\prime}=0}^{M-1}\left(\mathbf{B}_{m, m^{\prime}}\right) \mathbf{A}^{\prime} \mathbf{d}_{m^{\prime}}
$$

That is, (26) clearly shows that $\mathbf{B}$ multiplexes the signals $\mathbf{A}^{\prime} \mathbf{d}_{m^{\prime}}$ if $\left|\mathbf{B}_{m, m^{\prime}}\right|^{2} \neq 0$. Lastly, we highlight that the formulation of (25) is relevant because it allows a clear distinction in the roles of $\mathbf{A}^{\prime}$ and $\mathbf{B}$ in the context of EGC and equal reliability criterion (ERC), for designing robust communications systems.

\section{B. Equal Gain Criterion (EGC)}

As stated in [4], under the assumptions of CSI at the receiver only and a perfect feedback equalization (PFE), we have shown that the data symbols should experience the same channel gain in order to maximize the system's performance. In this paper, we term this condition as EGC. For the matrix of (25), a sufficient condition to guarantee the EGC is when $\left|\left(\mathbf{B} \otimes \mathbf{A}_{\mathrm{F}}^{\prime}\right)_{n, n^{\prime}}\right|^{2}=1 / N$ for all $\left(n, n^{\prime}\right)$, which has also been observed in [8]. The matrix $\mathbf{A}_{\mathrm{F}}^{\prime}=\mathbf{F}_{K} \mathbf{A}^{\prime}$ represents the modulation matrix per sub-block in FD. Clearly, the aforementioned condition is respected if $\left|\left(\mathbf{A}_{\mathrm{F}}^{\prime}\right)_{k, k^{\prime}}\right|^{2}=1 / K$ and $\left|(\mathbf{B})_{m, m^{\prime}}\right|^{2}=1 / M$ for all $\left(k, k^{\prime}\right)$ and $\left(m, m^{\prime}\right)$, respectively.

In summary, the matrix $\mathbf{A}^{\prime}$ is responsible for spreading the symbols in FD, while $\mathbf{B}$ is responsible for spreading the symbols among the sub-blocks.

\section{Equal Reliability Criterion (ERC)}

For the transmission without $\mathrm{CP}$, the data symbols suffer from interference as shown in (13), where the $m$-th sub-blocks is impaired by the interference term $\left(\mathbf{H}_{m-1}-\tilde{\mathbf{H}}_{m-1}\right) \mathbf{x}_{m-1}$ from the $(m-1)$-th sub-block. Thus, in addition to the equal-gain condition of [4], [14], it is expected that the most robust system will also have the data symbols affected by an equal level of interference, such that they are equally reliable, leading to the ERC. Because the interference is localized in time, the waveform should spread the symbols over time at a sub-block level in order to guarantee an equal level of interference to all symbols, which is attained by making $\left|(\mathbf{A})_{n, n^{\prime}}\right|^{2}=1 / K$ for all $\left(n, n^{\prime}\right)$ in $(25)$.

In this paper, we propose the BM-OCDM as a waveform that achieves the ERC, which is configured with $\mathbf{B}=\mathbf{F}_{M}$ and $\mathbf{A}_{\text {OCDM }}^{\prime}=\mathbf{F}_{K}^{\mathrm{H}} \boldsymbol{\Gamma}^{\mathrm{H}} \mathbf{F}_{K}$ in (25), where $\boldsymbol{\Gamma} \in \mathbb{C}^{N \times N}$ is a diagonal matrix whose elements are given by $\exp \left(j \pi n^{2} / K\right)$ for $n=0, \cdots, K-1$ [4], [19]. In FD, the OCDM per subblock is $\mathbf{A}_{\mathrm{OCDM}_{\mathrm{F}}}^{\prime}=\mathbf{F}_{K} \mathbf{A}_{\mathrm{OCDM}}^{\prime}=\boldsymbol{\Gamma}^{\mathrm{H}} \mathbf{F}_{K}$, which clearly achieves the EGC per sub-block due to $\left|\left(\boldsymbol{\Gamma}^{\mathrm{H}} \mathbf{F}_{K}\right)_{k, k^{\prime}}\right|$ for all $\left(k, k^{\prime}\right)$. Essentially, OCDM in FD is the SC waveform with phase rotation based on $\boldsymbol{\Gamma}$. Lastly, the requirement $\left|\left(\mathbf{A}_{\text {OCDM }}^{\prime}\right)_{n, n^{\prime}}\right|^{2}=1 / K$ for all $\left(n, n^{\prime}\right)$ is easily verified by noticing that $\mathbf{A}_{\text {OCDM }}^{\prime}$ is a circulant matrix whose first column has the diagonal elements of $\boldsymbol{\Gamma}$. Since BM-OCDM achieves the ERC and equal interference level, it is therefore an equally reliable waveform.

In the following, we provide an example to illustrate the unbalanced interference pattern for the CP-free system at subblock level. We consider $K=8$ and a channel with length $L=4$ samples and equal power taps with full CP-Restoration, which consists of copying the first $L-1$ samples of the $(m+$ 1)-th sub-block and adding them to the first $L-1$ samples of the $m$-th sub-block. With that, the received signal for the $m$-th sub-block after the full CP-restoration is given by

$$
\begin{aligned}
\mathbf{y}_{m} & =\tilde{\mathbf{y}}_{m}+\mathbf{G} \tilde{\mathbf{y}}_{m+1} \\
& =\mathbf{H}_{m} \mathbf{A}^{\prime} \mathbf{d}_{m}+\mathbf{H}_{m-1}^{\mathrm{p}} \mathbf{A}^{\prime} \mathbf{d}_{m-1} \\
& +\mathbf{G} \tilde{\mathbf{H}}_{m+1}^{\mathrm{n}} \mathbf{A}^{\prime} \mathbf{d}_{m+1}+\mathbf{w}_{m}+\mathbf{G} \mathbf{w}_{m+1},
\end{aligned}
$$

where $(\mathbf{G})_{n, n}=1$ for $n \leq L-1$ and zero for the remaining indexes, and $\mathbf{H}_{m-1}^{\mathrm{p}}=\left(\mathbf{H}_{m-1}-\tilde{\mathbf{H}}_{m-1}\right)$ is an upper triangular matrix based on (13). In (27), the term $\mathbf{H}_{m-1}^{\mathrm{p}} \mathbf{A}^{\prime} \mathbf{d}_{m-1}+\mathbf{H}_{m+1}^{\mathrm{n}} \mathbf{A}^{\prime} \mathbf{d}_{m+1}$ represents the interference from the adjacent sub-blocks and is localized in time due to the fact that $\mathbf{H}_{m-1}^{\mathrm{p}}$ is upper triangular and $\mathbf{G}$ only takes the first $L-1$ samples of the block $m+1$. The interference power per sample is given by $\boldsymbol{\Sigma}_{\text {int }_{m}}=$ $\operatorname{diag}\left(\mathbb{E}\left\{\mathbf{H}_{m-1}^{\mathrm{p}} \mathbf{H}_{m-1}^{\mathrm{p}^{\mathrm{H}}}\right\}+\mathbb{E}\left\{\mathbf{G} \tilde{\mathbf{H}}_{m+1} \tilde{\mathbf{H}}_{m+1}^{\mathrm{H}} \mathbf{G}\right\}\right)$, and is shown in the left side of Figure 2, where the first $L-1$ samples of the $m$-th sub-block are highly affected by the interference. The right graph of Figure 2 compares the signal to interference noise ratio (SINR) per data symbol for OCDM and SC under a zero forcing equalization ${ }^{4}$, which is computed as $\mathbf{1}_{K} / \operatorname{diag}\left(\mathbb{E}\left\{\mathbf{w}_{\mathrm{ZF}} \mathbf{w}_{\mathrm{ZF}}^{\mathrm{H}}\right\}\right)$. And the zero forcing noise is given

\footnotetext{
${ }^{4}$ The zero forcing equalizer is used here to make this example as simple as possible. The effect shown in the right graph of Figure 2 is also valid for other equalizers such as the MMSE of Section V.
} 
TABLE I

ERC: WAVEFORM CANDIDATES IN TIME AND FREQUENCY DOMAINS.

\begin{tabular}{l|c|c|l|l}
\hline waveform & FD, $\mathbf{A}_{\mathrm{F}}=\mathbf{B} \otimes \mathbf{F}_{K}^{\mathrm{H}} \mathbf{A}^{\prime}$ & $\mathrm{TD}, \mathbf{A}=\mathbf{B} \otimes \mathbf{A}^{\prime}$ & EGC & ERC \\
\hline OFDM & $\mathbf{I}_{M} \otimes \mathbf{I}_{K}$ & $\mathbf{I}_{M} \otimes \mathbf{F}_{K}{ }^{\mathrm{H}}$ & no & no \\
\hline SC & $\mathbf{I}_{M} \otimes \mathbf{F}_{K}$ & $\mathbf{I}_{M} \otimes \mathbf{I}_{K}$ & no & no \\
\hline OCDM & $\mathbf{I}_{M} \otimes \boldsymbol{\Gamma}_{K} \mathbf{F}_{K}$ & $\mathbf{I}_{M} \otimes \mathbf{F}_{K}^{\mathrm{H}} \boldsymbol{\Gamma}_{K} \mathbf{F}_{K}$ & no & no \\
\hline OTFS (BM-SC) & $\mathbf{F}_{M}^{\mathrm{H}} \otimes \mathbf{F}_{K}$ & $\mathbf{F}_{M}^{\mathrm{H}} \otimes \mathbf{I}_{K}$ & yes & no \\
\hline BM-OCDM & $\mathbf{F}_{M}^{\mathrm{H}} \otimes \boldsymbol{\Gamma}_{K} \mathbf{F}_{K}$ & $\mathbf{F}_{M}^{\mathrm{H}} \otimes \mathbf{F}_{K}^{\mathrm{H}} \boldsymbol{\Gamma}_{K} \mathbf{F}_{K}$ & yes & yes \\
\hline
\end{tabular}
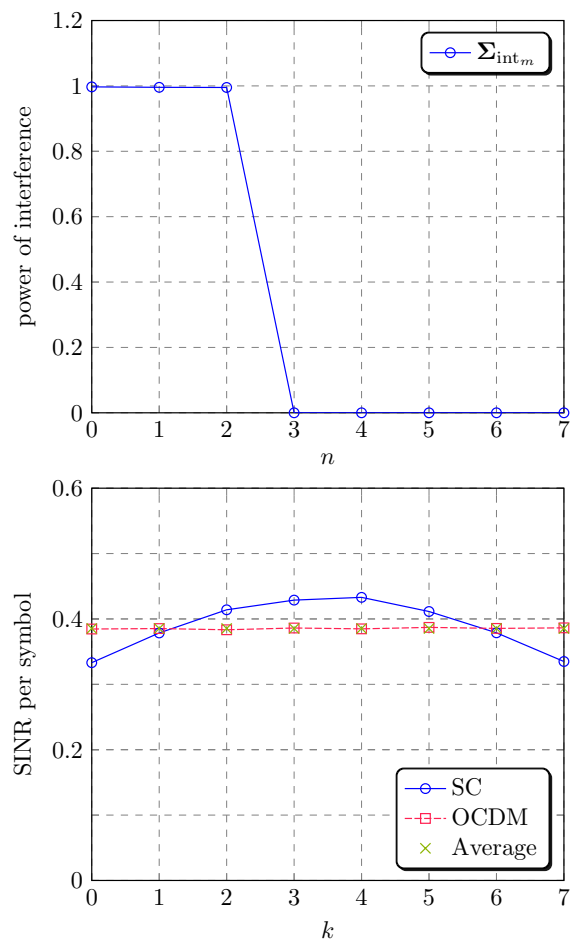

Fig. 2. Example of equal interference level due to waveform time spreading.

by $\mathbf{w}_{\mathrm{ZF}}=\left(\mathbf{H}_{m} \mathbf{A}^{\prime}\right)^{-1}\left(\mathbf{H}_{m-1}^{\mathrm{p}} \mathbf{A}^{\prime} \mathbf{d}_{m-1}+\mathbf{G} \tilde{\mathbf{H}}_{m+1}^{\mathrm{n}} \mathbf{A}^{\prime} \mathbf{d}_{m+1}+\right.$ $\left.\mathbf{w}_{m}+\mathbf{G w}_{m+1}\right)$. As expected, the SC waveform has different SINR levels per symbols due to time localization, indicating that they are not equally reliable. On the contrary, OCDM has the same interference level for all symbols.

\section{Waveform Candidates and Considerations}

In this subsection, we discuss waveforms without $\mathrm{CP}$, which are based on the modulation described in equation (25). These waveforms are listed in Table I.

In general, OFDM, SC and OCDM waveforms do not achieve the EGC because the symbols are localized in time at a sub-block level due to $\mathbf{B}=\mathbf{I}_{M}$. By consequence they also do not achieve the ERC. Due to frequency spreading of OCDM and SC, they outperform OFDM. In relation to OCDM and $\mathrm{SC}$, one can expect that OCDM outperforms SC because the interference pattern of adjacent sub-blocks in SC is unbalanced as shown in Subsection IV-C, i.e., the initial symbols of a given sub-block suffers most of the interference. On the other hand, OTFS (BM-SC) achieves the EGC because the symbols are also spread among the sub-blocks. However, for the same reason as SC, OTFS also has unbalanced interference and fails to achieve the ERC. In the OTFS case, although the symbols are spread among the sub-blocks, they are semi-localized in the time domain due to $\mathbf{A}^{\prime}=\mathbf{I}_{K}$ as shown in Table I. In the case of BM-OCDM, it achieves the EGC because the symbols are spread in frequency and also multiplexed among the sub-blocks. And it also attains the ERC because their data symbols are equally affected by the interference from the adjacent sub-blocks, due to time spreading. Since BM-OCDM is the only waveform that attains both ERC and EGC, it is expected to provide the best performance under the CP-free transmission scheme, that is eventually demonstrated by the numerical evaluation in Section VI.

\section{CP-Restoration AND EquaLIZATION}

We employ a similar receiver structure as in [5], [14]. In the following, we focus on the CP-Restoration and equalization employed in this work. The reader can find additional information in [5], [14], [20], [21] for the remaining receiver's components, namely, soft demapper, SISO decoder and soft mapper. In the subsequent derivations, we use $\boldsymbol{\mu}_{\mathbf{d}}^{\mathrm{a}} \in \mathbb{C}^{N}$ as the a-priori mean of the data and $\boldsymbol{\Sigma}_{\mathbf{d}}^{\mathrm{a}} \in \mathbb{C}^{N \times N}$ as the diagonal a-priori error variance of the data [5]. At the receiver's initial step, we set $\boldsymbol{\mu}_{\mathbf{d}}^{\mathrm{a}}=\mathbf{0}_{N}$ and $\boldsymbol{\Sigma}_{\mathbf{d}}^{\mathrm{a}}=\mathbf{I}_{N}$.

\section{A. CP-Restoration}

This operation is needed only for CP-free transmission and consists of copying the overlapping samples between the $m$ th and $(m+1)$-th sub-blocks. In this paper, we employ the partial CP-restoration method of [14], where the samples of the $(m+1)$-th sub-block are weighted and then added to the beginning of the $m$-th sub-block, in a way that the interference noise level is minimized by properly computing the weighting coefficients. Hence,

$$
\mathbf{y}_{m}=\left\{\begin{array}{ll}
\tilde{\mathbf{y}}_{m}+\mathbf{G}_{m} \tilde{\mathbf{y}}_{m+1}, & 0 \leq m \leq M-2 \\
\tilde{\mathbf{y}}_{m}+\mathbf{G}_{m} \tilde{\mathbf{y}}_{\mathrm{UW}_{1}}, & m=M-1
\end{array},\right.
$$

where $\mathbf{G}_{m} \in \mathbb{R}_{>0}^{K \times K}$ is the diagonal restoration matrix whose elements are derived in [14] as

$$
\begin{aligned}
& \left(\mathbf{G}_{m}\right)_{n, n}= \\
& \frac{\left(\hat{\mathbf{H}}_{m}^{\mathrm{p}}\right)_{n, *} \boldsymbol{\Sigma}_{\mathbf{x}_{m}}^{\mathrm{a}}\left(\hat{\mathbf{H}}_{m}^{\mathrm{p}}\right)_{n, *}^{\mathrm{H}}}{\left(\hat{\mathbf{H}}_{m}^{\mathrm{p}}\right)_{n, *} \boldsymbol{\Sigma}_{\mathbf{x}_{m}}^{\mathrm{a}}\left(\hat{\mathbf{H}}_{m}^{\mathrm{p}}\right)_{n, *}^{\mathrm{H}}+\left(\hat{\mathbf{H}}_{m}\right)_{n, *} \boldsymbol{\Sigma}_{\mathbf{x}_{m+1}}^{\mathrm{a}}\left(\hat{\mathbf{H}}_{m}\right)_{n, *}^{\mathrm{H}}+\sigma^{2}} .
\end{aligned}
$$

for $0 \leq m \leq M-2$. For the last sub-block $m=M-1$, we just set $\Sigma_{\mathbf{x}_{m+1}}^{\mathrm{a}}$ in (29) to zero because that represents perfect 
knowledge of the next signal, which is the UW sequence. In general, $\boldsymbol{\Sigma}_{\mathbf{x}_{m}}^{\mathrm{a}} \in \mathbb{R}^{K \times K}$ is a diagonal matrix whose elements are given by $\left(\boldsymbol{\Sigma}_{\mathbf{x}_{m}}^{\mathrm{a}}\right)_{n, n}=\left(\mathbf{A} \boldsymbol{\Sigma}_{\mathbf{d}}^{\mathrm{a}} \mathbf{A}^{\mathrm{H}}\right)_{n+m K+1, n+m K+1}$, for $n=0, \cdots, K-1$, and represents the error variance of the $m$-th sub-block signal. In addition, we consider the estimated channel matrix in (29) as $\left(\hat{\mathbf{H}}_{m}\right)_{n, n^{\prime}}=\hat{\mathbf{h}}_{m}\left[\left(n-n^{\prime}\right)_{K}\right]$ for $n, n^{\prime}=0, \cdots, K-1$, where $\hat{\mathbf{h}}_{m}=\mathbf{F}^{\mathrm{H}} \operatorname{diag}\left(\hat{\boldsymbol{\Lambda}}_{m}\right) \in \mathbb{C}^{K}$ is the estimated channel in time domain, and $\left(\hat{\mathbf{H}}_{m}^{\mathrm{p}}\right)_{n, n^{\prime}}=\left(\hat{\mathbf{H}}_{m}\right)_{n, n^{\prime}}$ for $n<n^{\prime}$ and zero otherwise. Notice that $\hat{\mathbf{H}}_{m}^{\mathrm{p}}$ is an upper triangular matrix and represents the channel interference from the previous sub-block in relation to the $m$-th sub-block [14].

\section{B. MMSE-PIC Equalizer}

In this subsection, we develop the generic component-wise conditionally unbiased (CWCU) minimum mean squared error with parallel interference cancellation (MMSE-PIC) receivers for any modulation matrix $\mathbf{A}$, considering the estimated channel with the errors defined in (22) and (23). We start by the CP-free transmission and follow similar steps as in [14]. We first define the received signal in FD as $\mathbf{Y}=$ $\left(\left(\mathbf{F} \tilde{\mathbf{y}}_{0}\right)^{\mathrm{T}}, \cdots,\left(\mathbf{F} \tilde{\mathbf{y}}_{M-1}\right)^{\mathrm{T}}\right)^{\mathrm{T}} \in \mathbb{C}^{N}$, which is a column vector whose elements are the concatenation of all received subblocks in FD. More precisely, we can write $\mathbf{Y}$ as

$$
\begin{aligned}
\mathbf{Y} & =\boldsymbol{\Lambda} \mathbf{A}_{\mathrm{F}} \mathbf{d}+\mathbf{W}_{\mathrm{CPR}}+\mathbf{W} \\
& =\hat{\boldsymbol{\Lambda}} \mathbf{X}-\boldsymbol{\Lambda}_{\mathrm{e}} \mathbf{X}+\mathbf{W}_{\mathrm{CPR}}+\mathbf{W}
\end{aligned}
$$

where $\mathbf{A}_{\mathrm{F}}=\left(\mathbf{I}_{M} \otimes \mathbf{F}_{K}\right) \mathbf{A}$ is the FD modulation matrix per sub-block. Also, we define $\mathbf{X}=\mathbf{A}_{\mathrm{F}} \mathbf{d}$ as the transmitted signal in FD per sub-block to keep the notation more compact. $\mathbf{W}$ is the FD noise per sub-block with power $\sigma^{2}$. The term $\mathbf{W}_{\mathrm{CPR}}=$ $\left(\mathbf{W}_{\mathrm{CPR}_{0}}^{\mathrm{T}}, \cdots, \mathbf{W}_{\mathrm{CPR}_{M-1}}^{\mathrm{T}}\right)^{\mathrm{T}}$ represents the concatenated FD interference after the $\mathrm{CP}$ restoration process, and the right most part of the last equation has decoupled the channel matrix $\boldsymbol{\Lambda}=$ $\hat{\boldsymbol{\Lambda}}-\boldsymbol{\Lambda}_{\mathrm{e}}$, where $\operatorname{diag}(\hat{\boldsymbol{\Lambda}})=\left(\operatorname{diag}\left(\hat{\boldsymbol{\Lambda}}_{0}\right)^{\mathrm{T}}, \cdots, \operatorname{diag}\left(\hat{\boldsymbol{\Lambda}}_{M-1}\right)^{\mathrm{T}}\right)^{\mathrm{T}}$ is the diagonal of estimated channel matrix, whose elements are obtained by concatenating the channel estimates in (17). The error matrix is obtained by concatenating the matrices of (21) as

$$
\boldsymbol{\Lambda}_{\mathrm{e}}=\left[\begin{array}{ccc}
\boldsymbol{\Lambda}_{\mathrm{e}_{0}} & & \\
& \ddots & \\
& & \boldsymbol{\Lambda}_{\mathrm{e}_{M-1}}
\end{array}\right]
$$

In the system model of (30), the channel error terms have a covariance matrix equal to

$$
\left(\boldsymbol{\Sigma}_{\mathrm{CH}}\right)_{k, k}=\left(\mathbb{E}\left\{\boldsymbol{\Lambda}_{\mathrm{e}} \mathbf{X X}^{\mathrm{H}} \boldsymbol{\Lambda}_{\mathrm{e}}^{\mathrm{H}}\right\}\right)_{k, k}=\left(\boldsymbol{\Sigma}_{\mathrm{CE}}\right)_{k, k}+\left(\boldsymbol{\Sigma}_{\mathrm{D}}\right)_{k, k}
$$

for $k=0, \cdots, N-1$, with its off-diagonal elements equal to zero. The channel estimation error and
Doppler error matrices are diagonal with elements respectively given by (22) and (23), resulting in $\operatorname{diag}\left(\boldsymbol{\Sigma}_{\mathrm{CE}}\right)=\left(\sigma_{\mathrm{CE}_{0,0}}^{2}, \sigma_{\mathrm{CE}_{0,1}}^{2}, \cdots, \sigma_{\mathrm{CE}_{M-1, K-1}}^{2}\right)^{\mathrm{T}}$ and $\operatorname{diag}\left(\boldsymbol{\Sigma}_{\mathrm{D}}\right)=\left(\sigma_{\mathrm{D}_{0,0}}^{2}, \sigma_{\mathrm{D}_{0,1}}^{2}, \cdots, \sigma_{\mathrm{D}_{M-1, K-1}}^{2}\right)^{\mathrm{T}}$. We highlight that there are no cross terms between the channel estimation and Doppler errors in (32), because their error matrices $\left(\boldsymbol{\Lambda}_{\mathrm{CEE}_{m}} \boldsymbol{\Lambda}_{\mathrm{DE}_{m}}^{\mathrm{H}}\right)_{k, k}=0 \forall k$ are diagonal and off-diagonal, respectively. Also, we obtain the right most side of (32) due to $\mathbb{E}\left\{\mathbf{X X}^{\mathrm{H}}\right\}=\mathbf{I}_{N}$. Finally, we remind the reader that $\mathbb{E}\left\{\boldsymbol{\Lambda}_{\mathrm{e}} \mathbf{X X}^{\mathrm{H}} \boldsymbol{\Lambda}_{\mathrm{e}}^{\mathrm{H}}\right\}$ is not diagonal in general. However, we neglect the off-diagonal elements for the sake of simplicity of the equalizer. This simplification is not expected to cause a significant performance loss. In fact, we have shown in [5] that taking the diagonal of the covariance matrix of (30) in the MMSE equalizer does not result in performance loss, which is the case of $\boldsymbol{\Sigma}_{\mathrm{CH}}$ defined in (32).

The interference per sub-block presented in (30) can be approximated by

$$
\begin{aligned}
\tilde{\mathbf{W}}_{\mathrm{CPR}_{m}} \approx \mathbf{F}_{K} & \left(\left(\mathbf{G}_{m}-\mathbf{I}\right) \hat{\mathbf{H}}_{m}^{\mathrm{p}} \mathbf{x}_{m}+\hat{\mathbf{H}}^{\mathrm{p}} \mathbf{x}_{m-1}\right. \\
& \left.+\mathbf{G}_{m} \hat{\mathbf{H}}_{m+1} \mathbf{x}_{m+1}+\mathbf{w}_{i}+\mathbf{G}_{m} \mathbf{w}_{m+1}\right)
\end{aligned}
$$

for $0 \leq m \leq M-2$. For $m=M-1$, the term $\mathbf{x}_{m+1}$ is replaced by $\mathbf{x}_{\mathrm{UW}}$ and the noise $\mathbf{w}_{m+1}$ is replaced by $\mathbf{w}_{\mathrm{UW}_{1}}$. The approximation in (33) neglects the channel error for simplicity. Again, it is expected that this simplification does not cause significant performance loss, because it will impact the computation of the interference power cause by the CP-Restoration. Assuming that the non-zero components of the estimated channel matrix $\mathbf{H}_{m}^{\mathrm{p}}$ is of orders of magnitude larger than its corresponding channel error matrix, the resulting interference power based on (33) should not deviate considerably from the true value. Also, it follows that

$$
\begin{aligned}
& \mathbb{E}\left\{\tilde{\mathbf{W}}_{\mathrm{CPR}_{m}}\right\} \approx \\
& \mathbf{F}_{K}\left(\left(\mathbf{G}_{m}-\mathbf{I}\right) \hat{\mathbf{H}}_{m}^{\mathrm{p}} \boldsymbol{\mu}_{\mathbf{x}_{m}}^{\mathrm{a}}+\hat{\mathbf{H}}_{m-1}^{\mathrm{p}} \boldsymbol{\mu}_{\mathbf{x}_{m-1}}^{\mathrm{a}}+\mathbf{G}_{m} \hat{\mathbf{H}}_{m+1} \boldsymbol{\mu}_{\mathbf{x}_{m+1}}^{\mathrm{a}}\right)
\end{aligned}
$$

For $0 \leq m \leq M-2$, and $m=M-1$, the term $\boldsymbol{\mu}_{\mathbf{x}_{m+1}}^{\mathrm{a}}$ is replaced by $\mathbf{x}_{\mathrm{UW}}$. Also, $\boldsymbol{\mu}_{\mathbf{x}_{m}}^{\mathrm{a}}=\left[\mathbf{A} \boldsymbol{\mu}_{\mathbf{d}}^{\mathrm{a}}\right]_{n=m N}^{(m+1) K-1}$ is the apriori average sub-block in time domain. In addition, we calculate the diagonal covariance matrix $\tilde{\mathbf{W}}_{m}$, in accordance with [14]

$$
\boldsymbol{\mu}_{\mathbf{d}}^{\mathrm{p}}=\boldsymbol{\mu}_{\mathbf{d}}^{\mathrm{a}}+\frac{\mathbf{A}_{\mathrm{F}}^{\mathrm{H}} \hat{\boldsymbol{\Lambda}}^{\mathrm{H}}\left(\hat{\boldsymbol{\Lambda}} \boldsymbol{\Sigma}_{\mathbf{X}}^{\mathrm{a}} \hat{\boldsymbol{\Lambda}}^{\mathrm{H}}+\boldsymbol{\Sigma}_{\mathrm{CH}}+\boldsymbol{\Sigma}_{\mathbf{W}_{\mathrm{CPR}}}+\sigma^{2} \mathbf{I}_{N}\right)^{-1}\left(\mathbf{Y}-\hat{\boldsymbol{\Lambda}} \boldsymbol{\mu}_{\mathbf{X}}^{\mathrm{a}}-\mathbb{E}\left\{\mathbf{W}_{\mathrm{CPR}}\right\}\right)}{\operatorname{diag}\left(\mathbf{A}_{\mathrm{F}}^{\mathrm{H}} \hat{\boldsymbol{\Lambda}}^{\mathrm{H}}\left(\hat{\boldsymbol{\Lambda}} \boldsymbol{\Sigma}_{\mathbf{X}}^{\mathrm{a}} \hat{\boldsymbol{\Lambda}}^{\mathrm{H}}+\boldsymbol{\Sigma}_{\mathrm{CH}}+\boldsymbol{\Sigma}_{\mathbf{W}_{\mathrm{CPR}}}+\sigma^{2} \mathbf{I}_{N}\right)^{-1} \hat{\boldsymbol{\Lambda}} \mathbf{A}_{\mathrm{F}}\right)}
$$


as

$$
\begin{aligned}
& \left(\boldsymbol{\Sigma}_{\left.\mathbf{W}_{\mathrm{CPR}_{m}}\right)_{k, k}}\right. \\
& \approx\left(\mathbb{E}\left\{\left(\mathbf{W}_{\mathrm{CPR}_{m}}-\mathbb{E}\left\{\mathbf{W}_{\mathrm{CPR}_{m}}\right\}\right)\left(\mathbf{W}_{\mathrm{CPR}_{m}}-\mathbb{E}\left\{\mathbf{W}_{\mathrm{CPR}_{m}}\right\}\right)^{\mathrm{H}}\right\}\right)_{k, k} \\
& \approx\left(\mathbf { F } _ { K } \left(\left(\mathbf{G}_{m}-\mathbf{I}\right) \hat{\mathbf{H}}_{m}^{\mathrm{p}} \boldsymbol{\Sigma}_{\mathbf{x}_{m}}^{\mathrm{a}} \hat{\mathbf{H}}_{m}^{\mathrm{p}^{\mathrm{H}}}\left(\mathbf{G}_{m}-\mathbf{I}\right)+\hat{\mathbf{H}}_{m-1}^{\mathrm{p}} \boldsymbol{\Sigma}_{\mathbf{x}_{m-1}}^{\mathrm{a}} \hat{\mathbf{H}}_{m}^{\mathrm{p}^{\mathrm{H}}}\right.\right. \\
& \left.\left.\quad+\mathbf{G}_{m} \hat{\mathbf{H}}_{m+1} \boldsymbol{\Sigma}_{\mathbf{x}_{m+1}}^{\mathrm{a}} \hat{\mathbf{H}}_{m+1}^{\mathrm{p}^{\mathrm{H}}} \mathbf{G}_{m}+\mathbf{G}_{m}^{2} \sigma^{2}\right) \mathbf{F}_{K}^{\mathrm{H}}\right)_{k, k},
\end{aligned}
$$

for $k=0, \cdots, K-1$. In the following, we incorporate the mean and error variance of $\tilde{\mathbf{W}}_{m}$ respectively as equation (36) in the bottom of previous page and

$$
\begin{aligned}
& \boldsymbol{\Sigma}_{\mathbf{d}}^{\mathrm{p}}=-\operatorname{diag}\left(\boldsymbol{\Sigma}_{\mathbf{X}}^{\mathrm{a}}\right) \\
& +\frac{\mathbf{1}_{N}}{\operatorname{diag}\left(\mathbf{A}_{\mathrm{F}}^{\mathrm{H}} \hat{\boldsymbol{\Lambda}}^{\mathrm{H}}\left(\hat{\boldsymbol{\Lambda}} \boldsymbol{\Sigma}_{\mathbf{X}}^{\mathrm{a}} \hat{\boldsymbol{\Lambda}}^{\mathrm{H}}+\boldsymbol{\Sigma}_{\mathrm{CH}}+\boldsymbol{\Sigma}_{\mathbf{W}_{\mathrm{CPR}}}+\sigma^{2} \mathbf{I}_{N}\right)^{-1} \hat{\boldsymbol{\Lambda}} \mathbf{A}_{\mathrm{F}}\right)}
\end{aligned}
$$

where $\mathbb{E}\left\{\mathbf{W}_{\mathrm{CPR}}\right\}=\mathbb{E}\left\{\left(\mathbf{W}_{\mathrm{CPR}_{0}}^{\mathrm{T}}, \cdots, \mathbf{W}_{\mathrm{CPR}_{M-1}}^{\mathrm{T}}\right)^{\mathrm{T}}\right\}$ is the concatenated noise plus interference of (34), and $\operatorname{diag}\left(\boldsymbol{\Sigma}_{\mathbf{W}_{\mathrm{CPR}}}\right)$ = $\left(\operatorname{diag}\left(\boldsymbol{\Sigma}_{\mathbf{W}_{\mathrm{CPR}_{0}}}\right)^{\mathrm{T}}, \cdots, \operatorname{diag}\left(\boldsymbol{\Sigma}_{\mathbf{W}_{\mathrm{CPR}_{M-1}}}\right)^{\mathrm{T}}\right)^{\mathrm{T}}$. In order to avoid the $N \times N$ matrix inversion in (36) and (37), the diagonal of the a-priori covariance matrix of the data in frequency domain is taken $\operatorname{as} \operatorname{diag}\left(\boldsymbol{\Sigma}_{\mathbf{X}}^{\mathrm{a}}\right)=\operatorname{diag}\left(\mathbf{A}_{\mathrm{F}} \boldsymbol{\Sigma}_{\mathbf{d}}^{\mathrm{a}} \mathbf{A}_{\mathrm{F}}^{\mathrm{H}}\right)$, with zeros in the off-diagonal elements. We have shown in [5] that this simplification causes no performance loss.

Lastly, we highlight that the system with $\mathrm{CP}$ does not require the $\mathrm{CP}$-Restoration procedure. In this case, the equalizer is obtained by making $\mathbb{E}\left\{\mathbf{W}_{\mathrm{CPR}}\right\}$ and $\operatorname{diag}\left(\boldsymbol{\Sigma}_{\mathbf{W}_{\mathrm{CPR}}}\right)$ equal to zero.

\section{Non-Iterative CP-Restoration}

One possible simplification of the receiver described above is to exclude the CP-restoration process in the MMSE-PIC structure of (36) and (37), by setting $\mathbb{E}\left\{\mathbf{W}_{\mathrm{CPR}}\right\}$ equal to zero and thus avoiding the computational burden of (34). In this case, $\operatorname{diag}\left(\boldsymbol{\Sigma}_{\mathbf{W}_{\mathrm{CPR}}}\right)$ should be computed only once. Obviously, this simplification is expected to result in decreased performance, which we evaluate in Section VI.

\section{NUMERICAL EVALUATION}

\section{A. Simulation Parameters}

In this subsection, we present the general parameters used in the numerical evaluation of subsections VI-B and VI-C. The PDP on the extended vehicular-A (EVA) model [21], with bandwidth of $B=4.32 \mathrm{MHz}$, resulting in the channel length of $L=12$ samples in (2). We assume $f_{\mathrm{c}}=5.9 \mathrm{GHz}$ as the center frequency, and a high mobility scenario with relative speed between TX and RX of $350 \mathrm{~km} / \mathrm{hr}$, which has a high maximum Doppler shift of $f_{\mathrm{d}}=1.92 \mathrm{kHz}$. The Doppler shift normalized to the subcarrier spacing (SCS) is $f_{\mathrm{d}_{\text {Norm }}}=$ $f_{\mathrm{d}} /(B / K)$. We define the energy overhead as the amount of samples used for CP and UW divided by the amount of samples of data, $N$, which for the $\mathrm{CP}$ and $\mathrm{CP}$-free systems are respectively computed as $E_{\mathrm{oh}_{\mathrm{CP}}}=\left(N_{\mathrm{CP}} M+N_{\mathrm{UW}}+N_{\mathrm{CP}}\right) / N$
TABLE II

Parameters of Channel I, $N=288$

\begin{tabular}{c|c|c|c|c|c}
\hline & $M=1$ & $M=2$ & $M=4$ & $M=6$ & $M=8$ \\
\hline FFT size, $K$ & 288 & 144 & 72 & 48 & 36 \\
$f_{\mathrm{d}_{\text {Norm }}}$ & 0.1275 & 0.0637 & 0.0319 & 0.0212 & 0.0159 \\
$E_{\text {oh }}$ & 0.25 & 0.32 & 0.46 & 0.6 & 0.74 \\
\hline
\end{tabular}

TABLE III

Parameters of Channel II, $N=576$

\begin{tabular}{c|c|c|c|c}
\hline & $M=2$ & $M=4$ & $M=6$ & $M=8$ \\
\hline FFT size, $K$ & 288 & 144 & 96 & 72 \\
$f_{\mathrm{d}_{\text {Norm }}}$ & 0.1275 & 0.0637 & 0.0425 & 0.0319 \\
$E_{\mathrm{oh}_{\mathrm{CP}}}$ & 0.16 & 0.23 & 0.3 & 0.37 \\
\hline
\end{tabular}

and $E_{\mathrm{oh}}=\left(N_{\mathrm{CP}}+N_{\mathrm{UW}}\right) / N$. Thus, considering unitary power for the channel, the average energy per encoded symbols is $E_{\mathrm{S}}=1+E_{\mathrm{oh}}$ and $E_{\mathrm{S}}=1+E_{\mathrm{oh}}$ for $\mathrm{CP}$ and $\mathrm{CP}$-free systems, respectively. We set the $\mathrm{CP}$ size as $N_{\mathrm{CP}}=20$ and the UW size is $N_{\mathrm{UW}}=32$. Finally, we define two set of configurations, namely, Channel I for $N=288$ and Channel II for $N=576$. The parameters of Channels I and II regarding the normalized Doppler and energy overhead are summarized in Tables (II) and (III).

The link level performance is assessed in terms of frame error rate (FER). As in [4], [14], we employ an recursive systematic convolutional (RSC) encoder with BCJR log-MAP decoder with rate $R=1 / 2$ and generating polynomial $\{133,171\}_{8}$. A higher code rate of $R=3 / 4$ is obtained by puncturing $66 \%$ of the parity bits. For the CP-free systems, we consider the schemes with and without iterative $\mathrm{CP}$ Restoration of Section V, which are named $I \times \mathrm{CP}-$ Restoration and $1 \times \mathrm{CP}$-Restoration, respectively. A maximum number of iterations of the equalizer is set to $I=7$. The energy per bit is defined as $E_{\mathrm{b}}=E_{\mathrm{S}} /\left(R \log _{2} J\right)$, where $E_{\mathrm{S}}$ is the uncoded symbol energy, with $J=4$ and $J=16$ for QPSK and 16QAM, respectively.

\section{B. Frame Optimization and $C P$ vs CP-free FER Comparison}

The goals of this subsection are i) to validate the equations (22) and (23) by comparing the averaged theoretical expressions with simulation, ii) to demonstrate the frame optimization by numerically minimizing the composite channel errors in (24), and iii) to validate the employment of the CP-free scheme by comparing it to the regular system with $\mathrm{CP}$.

The first evaluation depicted in the top graph of Figure 3 compares the averaged CEE and DE of (24) against simulation the Channel I of Table II. As in (24), we denote the averaged $\mathrm{CEE}$ and $\mathrm{DE}$ respectively as $\sigma_{\mathrm{CE}_{\mathrm{CP}}}^{2}(M)=$ $1 /(M \cdot K) \sum_{m=0}^{M-1} \sum_{k=0}^{K-1} \sigma_{\mathrm{CE}_{m, k}}^{2}$ and $\sigma_{\mathrm{D}}^{2}(M)=1 /(M \cdot$ K) $\sum_{m=0}^{M-1} \sum_{k=0}^{K-1} \sigma_{\mathrm{D}_{m, k}}^{2}$. The results show agreement between theory and experiment, therefore demonstrating that the theoretical development of Section III is correct. Moreover, $M=1$ has the highest DE because $f_{\mathrm{d}_{\mathrm{Norm}}}=0.1275$ is highest according to Table II. In this case, the sub-block transmission 

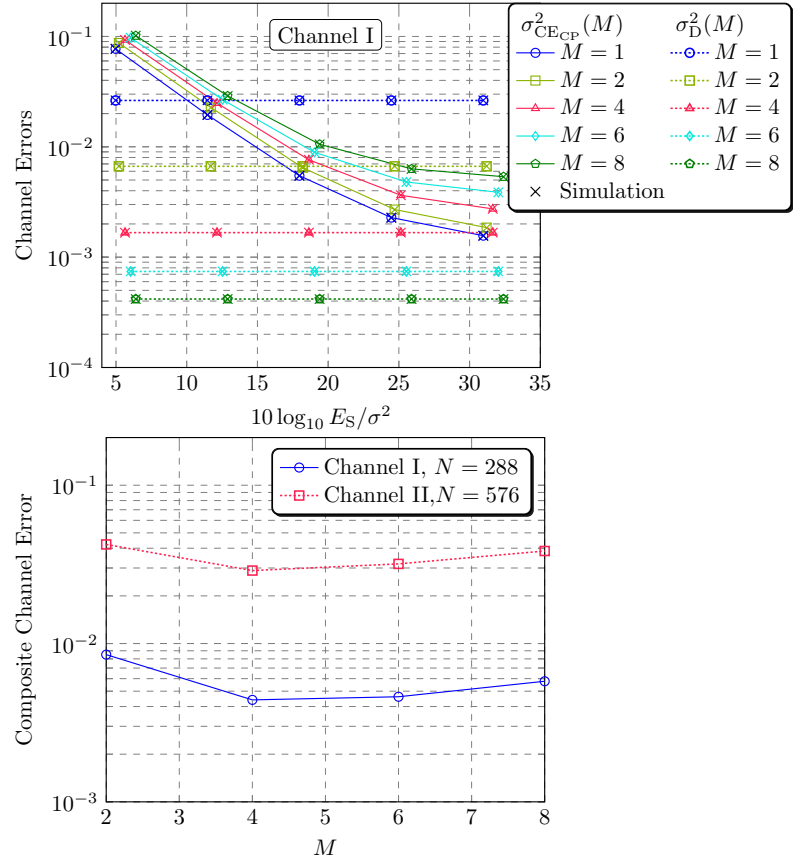

Fig. 3. Evaluation of errors. Left graph: comparison between CEE and DE for Channel I. Right graph: composite channel error for Channels I and II.

takes the longest time, i.e., $K=288$ samples. Thus, the channel varies considerably throughout the data transmission and causes high ICI. As we increase $M, \sigma_{\mathrm{D}}^{2}(M)$ is decreased as the transmission time of a sub-block also decreases. On the other hand, making $M$ larger increases the temporal distance between the two UWs, because each sub-block has its own $\mathrm{CP}$, which results in an increase of $\sigma_{\mathrm{CE}_{\mathrm{CP}}}^{2}(M)$, i.e., channel estimation becomes less accurate.

The bottom graph of Figure 3 numerically computes the minimization of $\sigma_{\mathrm{CE}}^{2}(M)+\sigma_{\mathrm{D}}^{2}(M)$ over $M$ as stated in (24), where the highest $E_{\mathrm{S}} / \sigma^{2}$ points of the left graph are considered. First, we observe that composite channel error is a convex function, which is expected due to the trade-off between $\sigma_{\mathrm{CE}}^{2}{ }_{\mathrm{CP}}(M)$ and $\sigma_{\mathrm{D}}^{2}(M)$ that has been discussed in the last paragraph and in Subsection III-C. Also, one observes that $M=4$ provides the lowest error for both channels I and II, which therefore should provide the best FER performance at this $E_{\mathrm{S}} / \sigma^{2}$ region.

In order to validate the result of the last paragraph in terms of performance, the FER of CP-BM-OCDM with $R=1 / 2$ is investigated for different values $M$, whose outcomes are depicted in the top graph of Figure 4. In particular, the system with $M=4$ provides the best performance for the high SNR region of the 16-QAM curves, as expected. For the QPSK curves, the system with $M=2$ and $M=4$ have similar performance, because the composite channel error in the smaller SNR region is similar for both configurations. Therefore, one should note that the modulation and coding scheme (MCS) plays a role on the frame optimization because it dictates the SNR region of operation. Furthermore, as we have mentioned in Subsection III-C, the minimization approach of (24) is not directly applicable to the CP-free transmission. Thus, in the bottom graph of Figure 4 we compute the FER for BM-OCDM
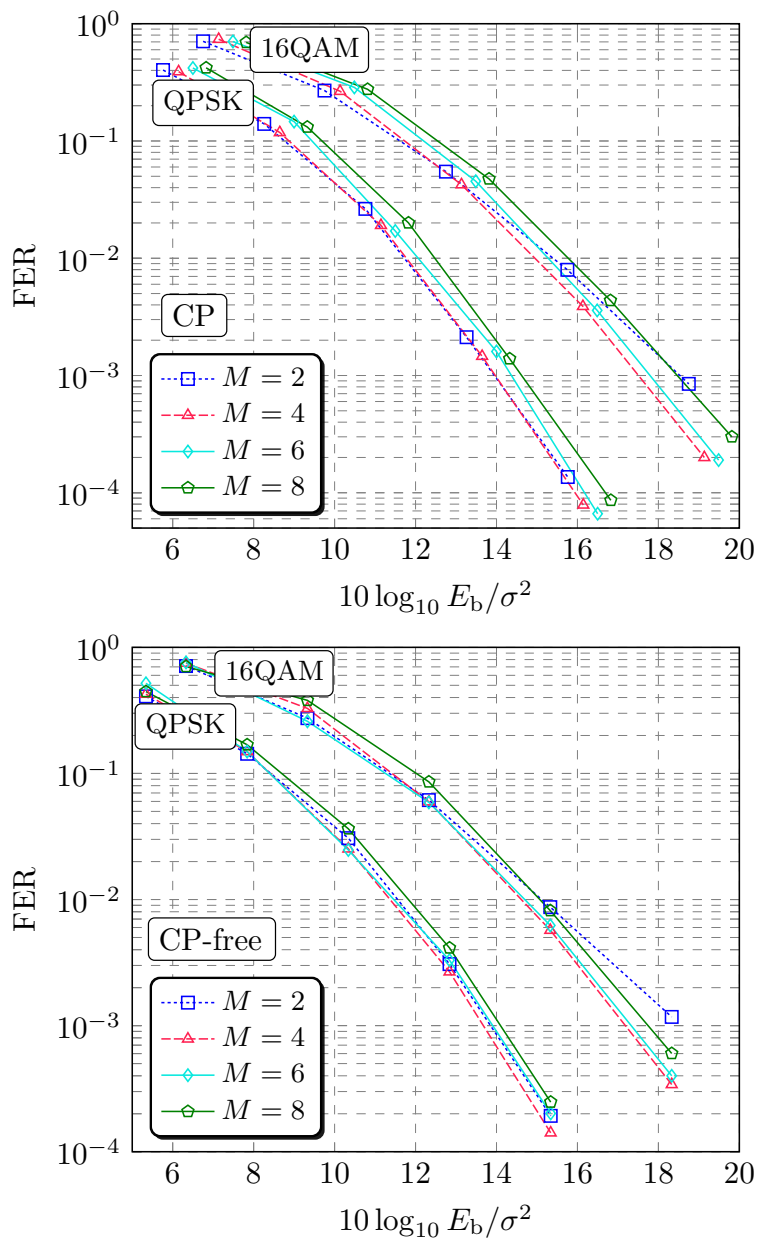

Fig. 4. FER evaluation of CP-BM-OCDM (CP) and BM-OCDM (CP-free) with different $M$ for Channel I.

without CP for different $M$, where the configuration $M=4$ presents again the best performance for the high SNR region. It is worth highlighting that this outcome is not assured to be general, i.e., it is not possible to affirm that the best $M$ for the $\mathrm{CP}$ system is always the same for the CP-free system. A more systematic investigation in this regard is necessary in order to draw more general conclusions.

Lastly, we recall that the motivation in designing a $\mathrm{CP}$-free system is to improve the $\mathrm{CE}$ because the lack of $\mathrm{CP}$ reduces the overall transmission time. For this purpose, the top and middle graphs of Figure 5 compares the averaged CEE for $\mathrm{CP}$ and CP-free systems, defined as $\sigma_{\mathrm{CE}_{\mathrm{CP}}}^{2}(M)$ and $\sigma_{\mathrm{CE}}^{2}(M)$, respectively. As expected, the outcomes confirm that $\sigma_{\mathrm{CE}}^{2}(M)$ does not increase considerably with increasing $M$ in relation to $\sigma_{\mathrm{CE}}^{2}(M)$. However, we highlight that the results of Figure 5 is not sufficient to determine the performance improvement due to additional interference. Therefore, the bottom graph of Figure 5 evaluates $\mathrm{BM}-\mathrm{OCDM}$ with $\mathrm{CP}$ and without $\mathrm{CP}$ for $M=4$ under both Channels I and II. The CP-free system employs the $I \times \mathrm{CP}$-Restoration receiver. The outcomes reveal that the CP-free transmission provides better FER results under both Channels I and II, indicating that the iterative receiver of BM-OCDM can significantly reduce the interference power of 

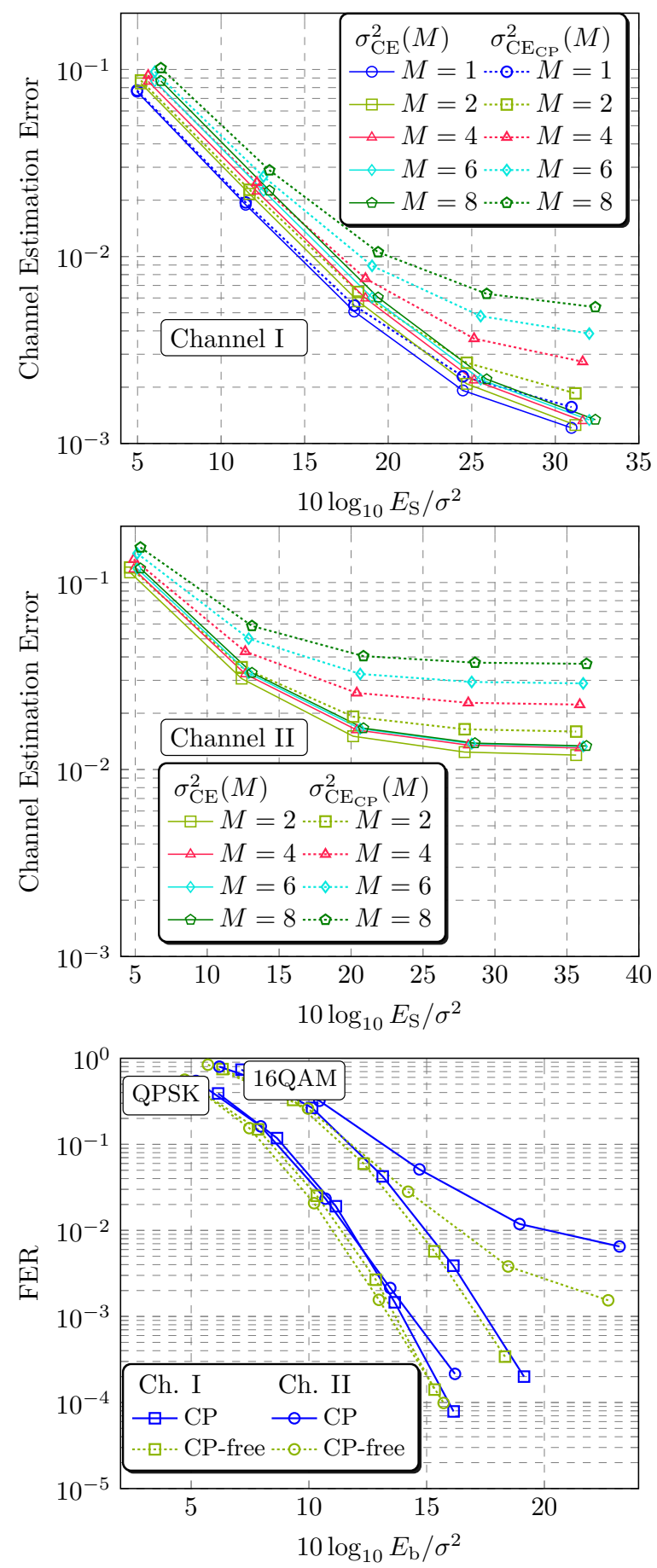

Fig. 5. Left and middle graphs: channel estimation errors for the transmission with and without $\mathrm{CP}$. Right graph: $\mathrm{CP}$ and $\mathrm{CP}$-free comparison with $M=4$ for Channels I and II.

(35). Also, we note that the improvement is more accentuated for system under Channel II, which happens because this configuration has a higher CEE. Thus, improving the channel estimation by the CP-free system has a higher impact.

In summary, in this subsection we have demonstrated how decoupling the channel errors into CEE and DE leads to the frame optimization by adjusting $K$ and $M$. In addition, we have compared the channel errors for the $\mathrm{CP}$ and $\mathrm{CP}$-free systems, showing that the CE is indeed improved when the
CP-free system is employed. Lastly, we have validated the employment of CP-free system in terms of FER, which has shown superior performance than the respective CP system.

\section{Waveform Comparison}

In this subsection, the waveform candidates of Subsection IV-D are compared for the CP-free systems. We consider the systems with $M=4$ for both Channels I and II, leading to $(K, M)=(72,4)$ and $(K, M)=(144,4)$, respectively. The remaining parameters are described in Subsection VI-A.

The left graphs of Figure 6 depict the results for the systems employing the $I \times \mathrm{CP}$-Restoration receiver with $R=1 / 2$ code rate. First of all, we observe the superior performance of the spreading waveforms compared to OFDM due to the spreading, which we have already reported in [4], [5]. Another consideration to make is that Channel I is not time-selective enough in order for BM-OCDM and OTFS to demonstrate considerable improvement in relation to OCDM and SC. Conversely, for Channel II, we observe that the BM waveforms have a better performance compared to the conventional ones, which can be explained by the fact that the channel is more time-selective. In addition, the spreading waveforms provide similar performance, where only a minor improvement of BMOCDM in relation to OTFS is observed for Channel II and 16QAM. This result indicates that the iterative CP-Restoration is effective also for SC and OTFS. In other words, the decoder is able to produce feedback soft bits which are accurate enough to reconstruct the $\mathrm{CP}$, such that the residual interference is not significant to cause a large performance deviation between BM-OCDM and OTFS. Since the decoder capability depends on the code rate, the middle graphs of Figure 6 investigate the performance of BM-OCDM and OTFS for $R=3 / 4$ using the iterative CP-Restoration, where the respective systems with $\mathrm{CP}$ are evaluated as a benchmark. For Channel I, the QPSK results behave differently from the 16-QAM. That is, the CPfree system with QPSK outperforms the CP system, which does not happen with the 16-QAM. As one could infer, this outcome reveals that the MCS impacts the effectiveness of the CP-Restoration method. In particular, this outcome can be explained by the fact that a system with higher code rate has a weaker code, meaning that the feedback from the decoder is less accurate which negatively impacts the CP-Restoration. For Channel II, a better performance of the CP-free system for both QPSK and 16-QAM is observed. Due to the higher residual interference, a perceptible performance gap between BM-OCDM and OTFS is seen which is expected based on the ERC of Section IV. Lastly, an impractical FER higher than $10^{-2}$ is observed for Channel II and 16-QAM. An obvious approach to decrease the FER is to employ multiple antennas for spatial diversity, which is proposed as future work.

Next, we analyze the right graphs of Figure 6, which show the receiver without the iterative CP-Restoration. Firstly, the OTFS system with QPSK perform similarly to BM-OCDM, indicating that the residual interference is not high enough to provoke a considerable performance gap with this MCS. This outcome changes with 16-QAM. In this case, a significant performance loss in OTFS is verified, showing that higher MCS are more susceptible to the interference. 

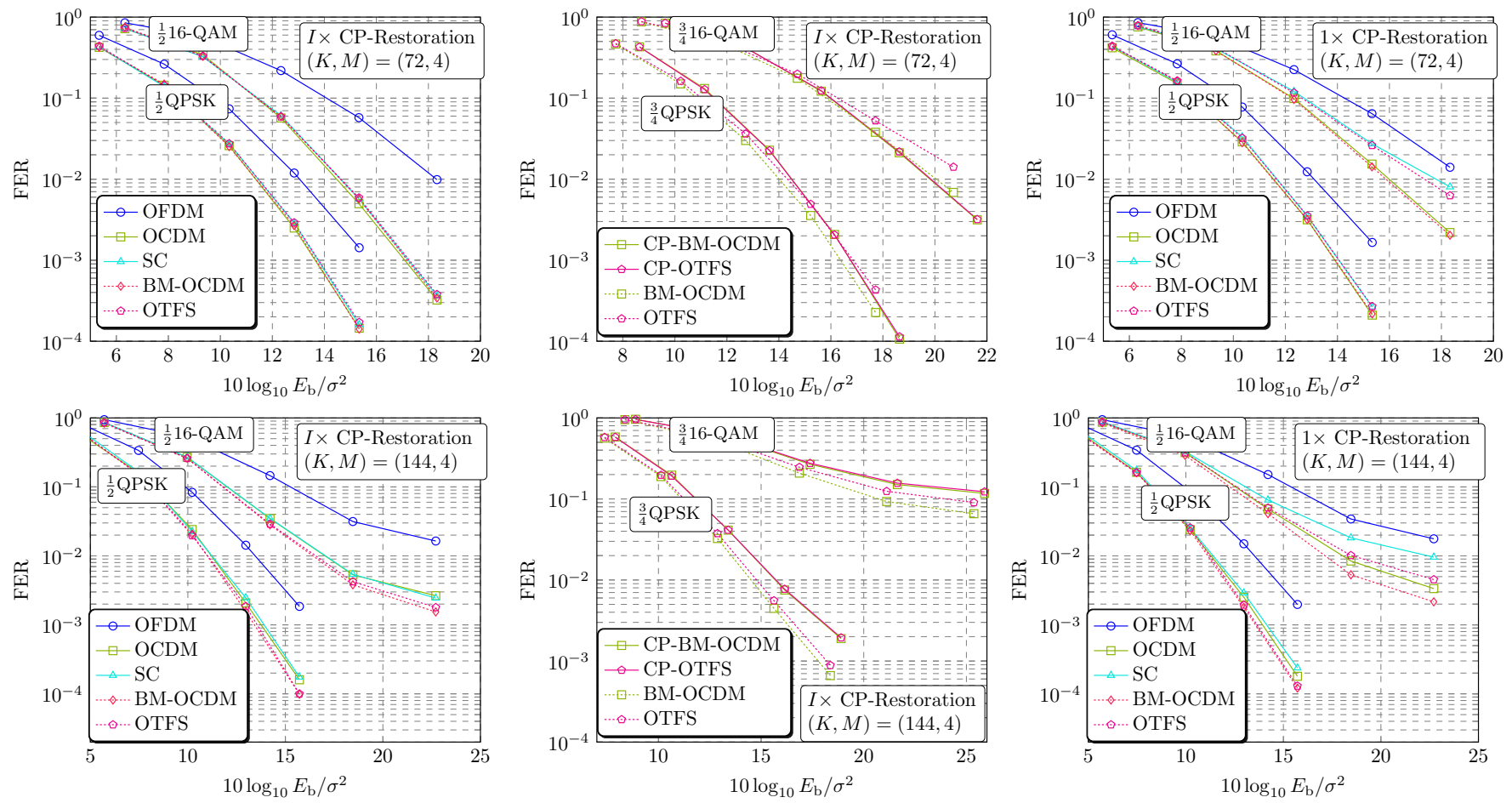

Fig. 6. FER of waveforms without $\mathrm{CP}$ for the receiver with and without iterative CP-Restoration, for $(K, M)=(72,4)$.

In conclusion, this subsection has compared the proposed BM-OCDM waveform with OTFS. The outcomes revealed that BM-OCDM is advantageous against OTFS depending on the MCS and receiver design. When the iterative CPRestoration receiver is employed with code rate $R=3 / 4$, the performance gap is noticed. For the non iterative CPRestoration, the performance gap is observed for 16-QAM.

\section{CONCLUSION}

With the goal of providing a robust wireless communication framework for highly doubly-dispersive channels, we have jointly studied three different concepts, namely, i) channel estimation, ii) transmission without $\mathrm{CP}$ among the sub-blocks to improve channel estimation, and iii) waveform design based on the equal reliability criterion. For the channel estimation and frame design part, we provided a novel analysis for the channel related errors where we can minimize the overall error by using the correct number of sub-blocks. To this end, we have decoupled the channel related errors in two parts, namely, channel estimation error (CEE) and Doppler error (DE). We have provided theoretical expressions for both errors and established a trade-off that arises from the frame configuration. For the numerical evaluation, we have used an optimized frame setting with relative speed between transmitter and receiver of $350 \mathrm{~km} / \mathrm{h}$ and bandwidth $B=4.32 \mathrm{MHz}$ for EVA channel PDP. In addition, we have also considered CPfree transmission in order to improve channel estimation at the cost of extra signal processing to deal with the interblock interference. Furthermore, we have proposed a lowcomplexity implementation, where the $\mathrm{CP}$-restoration process is not included in the iterative receiver. Lastly, we have provided a waveform design based on the equal reliability criterion (ERC) for the CP-free waveforms. That is, we have proposed a waveform which spreads the symbols over all time and frequency instances such that it not only targets the equal channel gain condition due to time and frequency selectivity, but also has equally distributed interference such that all symbols experience the same interference level and are equally reliable. We have shown that block multiplexing orthogonal chirp division multiplexing (BM-OCDM) attains the ERC and provides the best link-level performance in general. The outcomes revealed that BM-OCDM is advantageous against OTFS depending on the MCS and receiver design. When the iterative CP-Restoration receiver is employed with code rate $R=3 / 4$, the performance gap is noticed. For the non iterative CP-Restoration, the performance gap is observed for 16-QAM.

A natural follow-on from this work is to extend the schemes to a MIMO setting. The channel estimation part can be easily realized by considering shifted Zadoff-Chu sequences from different antennas. Another aspect to be investigated is lowcomplexity channel estimation, where our generic closedform model can serve as a benchmark for future research, e.g., investigations on the performance loss of low-complexity channel estimation schemes. Lastly, since in this paper a perfect synchronized system has been assumed, the impact of time and frequency offset in the performance is also relevant for future work. In particular, time offset can impact the effectiveness of the CP-Restoration process and penalizes OTFS more than BM-OCDM. 


\section{APPENDIX A \\ INTERPolation COEFFICIENTS $\mathbf{C}_{m, k}$}

We provide analytical solution to compute the coefficients $\mathbf{C}_{m, k}$ in (18) based on the model of II-B. In general, the solution for the coefficients under the MSE criterion are given by [13]

$$
\mathbf{C}_{m, k}=\mathbb{E}\left\{\left(\boldsymbol{\Lambda}_{m}\right)_{k, k} \hat{\mathbf{P}}_{k}^{\mathrm{H}}\right\} \mathbb{E}\left\{\hat{\mathbf{P}}_{k} \hat{\mathbf{P}}_{k}^{\mathrm{H}}\right\}^{-1} .
$$

Therefore, we need to compute the expectations $\mathbb{E}\left\{\hat{\mathbf{P}}_{k} \hat{\mathbf{P}}_{k}^{\mathrm{H}}\right\} \quad$ and $\quad \mathbb{E}\left\{\left(\boldsymbol{\Lambda}_{m}\right)_{k, k} \hat{\mathbf{P}}_{k}^{\mathrm{H}}\right\}$. Recalling that $\hat{\mathbf{P}}_{k}=\left(\left(\hat{\boldsymbol{\Lambda}}_{\mathrm{UW}_{0}}\right)_{k},\left(\hat{\boldsymbol{\Lambda}}_{\mathrm{UW}_{1}}\right)_{k}\right)^{\mathrm{T}}$, one can directly observe that

$$
\begin{aligned}
& \mathbb{E}\left\{\hat{\mathbf{P}}_{k} \hat{\mathbf{P}}_{k}^{\mathrm{H}}\right\}= \\
& {\left[\begin{array}{cc}
\mathbb{E}\left\{\left|\left(\hat{\boldsymbol{\Lambda}}_{\mathrm{UW}_{0}}\right)_{k}\right|^{2}\right\} & \mathbb{E}\left\{\left(\hat{\boldsymbol{\Lambda}}_{\left.\left.\mathrm{UW}_{0}\right)_{k}\left(\hat{\boldsymbol{\Lambda}}_{\mathrm{UW}_{1}}\right)_{k}^{\mathrm{H}}\right\}}\right.\right. \\
\mathbb{E}\left\{\left(\hat{\boldsymbol{\Lambda}}_{\mathrm{UW}_{1}}\right)_{k}\left(\hat{\boldsymbol{\Lambda}}_{\mathrm{UW}_{0}}\right)_{k}^{\mathrm{H}}\right\} & \mathbb{E}\left\{\left|\left(\hat{\boldsymbol{\Lambda}}_{\mathrm{UW}_{0}}\right)_{k}\right|^{2}\right\}
\end{array}\right]}
\end{aligned}
$$

has the power of the estimated $k$-th carrier of the channel in its diagonal and the correlation between the two channels estimated by the different UWs in the off diagonal. To solve this equation in closed form, we rewrite the estimated UW channel in (15) as

$$
\begin{aligned}
\hat{\mathbf{\Lambda}}_{\mathrm{UW}_{u}} & =\mathbf{F}_{K} \mathbf{D U}^{-1} \mathbf{y}_{\mathrm{UW}_{u}} \\
& =\mathbf{F}_{K} \mathbf{D} \mathbf{U}^{-1}\left(\tilde{\mathbf{H}}_{\mathrm{UW}_{u}} \mathbf{x}_{\mathrm{UW}}+\mathbf{w}_{\mathrm{UW}_{u}}\right),
\end{aligned}
$$

where the inverse of the matrix $\mathbf{U}=\mathbf{F}_{\mathrm{UW}}^{\mathrm{H}} \operatorname{diag}\left\{\mathbf{X}_{\mathrm{UW}}\right\} \mathbf{F}_{\mathrm{UW}}$ is essentially performing the operations of (14) and the inner parenthesis of (15) at once for compactness. And the second line of (40) is taken from equations (6) and (7). Finally, we note that because $\mathbf{X}_{\mathrm{UW}}^{-1}=\mathbf{X}_{\mathrm{UW}}^{\mathrm{H}}$, we have that $\mathbf{U}^{-1}=\mathbf{U}^{\mathrm{H}}$.

The general closed form solution for all elements of $\mathbb{E}\left\{\hat{\mathbf{P}}_{k} \hat{\mathbf{P}}_{k}^{\mathrm{H}}\right\}$ is given in (41). The first line $(a)$ is the correlation matrix of the signal in (40) for an arbitrary combination of $u$ and $u^{\prime}$, where the first and last UWs are represented by the indexes 0 and 1 , respectively. The line $(b)$ follows by considering that the expectation operator is restricted only to the channel matrices and the AWGN noise is zero mean and uncorrelated for $u \neq u^{\prime}$, which is described by the use of the delta function $\delta[\cdot]$. Also, the noise is uncorrelated to the channel, thus there are no cross terms between the noise and the channel. In line $(c)$, solve the remaining expectation operation with the matrix $\mathbf{R}\left(u, u^{\prime}\right) \in \mathbb{C}^{N_{\mathrm{UW}} \times N_{\mathrm{Uw}}}$, given by

$$
\begin{aligned}
& \left(\mathbf{R}\left(u, u^{\prime}\right)\right)_{n, n^{\prime}} \\
& \stackrel{(a)}{=} \mathbb{E}\left\{\left(\tilde{\mathbf{H}}_{\mathrm{UW}_{u}}\right)_{n, *} \mathbf{x}_{\mathrm{UW}} \mathbf{x}_{\mathrm{UW}}^{\mathrm{H}}\left(\tilde{\mathbf{H}}_{\mathrm{UW}_{u^{\prime}}}\right)_{n^{\prime}, *}^{\mathrm{H}}\right\} \\
& \stackrel{(b)}{=} \operatorname{Tr}\left\{\left(\mathbf{x}_{\mathrm{UW}} \mathbf{x}_{\mathrm{UW}}^{\mathrm{H}}\right) \mathbb{E}\left\{\left(\tilde{\mathbf{H}}_{\mathrm{UW}_{u^{\prime}}}\right)_{n^{\prime}, *}^{\mathrm{H}}\left(\tilde{\mathbf{H}}_{\mathrm{UW}_{u}}\right)_{n, *}\right\}\right\} \\
& \stackrel{(c)}{=} \operatorname{Tr}\left\{\left(\mathbf{x}_{\mathrm{UW}} \mathbf{x}_{\mathrm{UW}}\right)^{\mathrm{H}} \operatorname{shift}_{n^{\prime}, n}\left\{\boldsymbol{\Sigma}_{\mathbf{H}_{\mathrm{UW}}}\left(n-n^{\prime}, u-u^{\prime}\right)\right\}\right\} .
\end{aligned}
$$

The line $(a)$ is simply the equivalence taken from $(41)(c)$. Line $(b)$ is obtained using the relation [22] $\mathbb{E}\left\{\mathbf{v}_{\mathbf{0}} \mathbf{Q} \mathbf{v}_{\mathbf{1}}{ }^{\mathrm{H}}\right\}=$ $\operatorname{Tr}\left\{\mathbf{Q E}\left\{\mathbf{v}_{1}^{\mathrm{H}} \mathbf{v}_{0}\right\}\right\}$, where $\mathbf{v}_{\mathbf{0}}, \mathbf{v}_{\mathbf{1}}$ are zero-mean row random vectors with size $K$ and $\mathbf{Q}$ is a constant hermitian matrix with size $K \times K$. In the case of (42), $\mathbf{x}_{\mathrm{UW}} \mathbf{x}_{\mathrm{UW}}^{\mathrm{H}}$ is by definition hermitian, thus we obtained the element $\left(\mathbf{R}\left(u, u^{\prime}\right)\right)_{n, n^{\prime}}$ for all $n, n^{\prime}=0, \cdots, N_{\mathrm{UW}}-1$. Finally, in the line $(c)$ of (42) we have the term

$$
\boldsymbol{\Sigma}_{\mathbf{H}_{\mathrm{UW}}}\left(\Delta_{n}, \Delta_{u}\right)\left[\begin{array}{cccc}
\rho_{0} & & & \\
& 0 & & \\
& & \ddots & \\
& & & \rho_{1}
\end{array}\right] \Upsilon\left(\Delta_{n}+\Delta_{u} \Delta_{\mathrm{UW}}\right),
$$

whose entries depend on the channel's PDP. One can easily check this arrangement by taking the first row of the first UW channel $\tilde{\mathbf{H}}_{\mathrm{UW}_{0}}$ from equations (8) and (9) as an example, i.e., $n=n^{\prime}=u=u^{\prime}=0$. In addition, the $\operatorname{shift}_{n^{\prime}, n}\{\cdot\}$ operation is necessary to correct the channel coefficients' positions in (43). For instance, if $n=0$ and $n^{\prime}=1$, the correlation matrix will be the one of (43) with a row shift by one position. Similarly, changing $n$ will result in column shift. Also, the correlation term $\Upsilon\left(\Delta_{n}+\Delta_{u} \Delta_{\mathrm{UW}}\right)=$ $\Upsilon\left(n+u \Delta_{\mathrm{UW}}-\left(n^{\prime}+u^{\prime} \Delta_{\mathrm{UW}}\right)\right)$ takes into account the temporal correlation between channel responses at the time indexes $n+u \Delta_{\mathrm{UW}}$ and $n^{\prime}+u^{\prime} \Delta_{\mathrm{UW}}$. Therefore, by plugging equation (41) into (39), we have the solution in equation (44). Since we are interested in the diagonal of (41), we take the element $(\cdot)_{k, k}$ Finally, the remaining noise term can be simplified to $\sigma^{2} L / N$ such as in [13].

In order to compute $\mathbb{E}\left\{\left(\boldsymbol{\Lambda}_{m}\right)_{k, k} \hat{\mathbf{P}}_{k}^{\mathrm{H}}\right\}$, we note that $\operatorname{diag}\left(\boldsymbol{\Lambda}_{m}\right)=\operatorname{diag}\left(\mathbf{F} \overline{\mathbf{H}}_{m} \mathbf{F}^{\mathrm{H}}\right)$ is the averaged channel impulse response in FD, as stated in Equation (19), where $\overline{\mathbf{H}}_{m} \in \mathbb{C}^{K \times K}$ is a circulant matrix whose first column is the

$$
\begin{aligned}
& \mathbb{E}\left\{\hat{\boldsymbol{\Lambda}}_{\mathrm{UW}_{u}} \hat{\boldsymbol{\Lambda}}_{\mathrm{UW}_{u^{\prime}}}^{\mathrm{H}}\right\} \stackrel{(a)}{=} \mathbb{E}\left\{\mathbf{F}_{K} \mathbf{D} \mathbf{U}^{\mathrm{H}}\left(\tilde{\mathbf{H}}_{\mathrm{UW}_{u}} \mathbf{x}_{\mathrm{UW}}+\mathbf{w}_{\mathrm{UW}_{u}}\right)\left(\tilde{\mathbf{H}}_{\mathrm{UW}_{u^{\prime}}} \mathbf{x}_{\mathrm{UW}}+\mathbf{w}_{\mathrm{UW}_{u^{\prime}}}\right)^{\mathrm{H}} \mathbf{U D}^{\mathrm{H}} \mathbf{F}_{K}^{\mathrm{H}}\right\} \\
& \stackrel{(b)}{=} \mathbf{F}_{K} \mathbf{D} \mathbf{U}^{\mathrm{H}} \mathbb{E}\left\{\tilde{\mathbf{H}}_{\mathrm{UW}_{u}} \mathbf{x}_{\mathrm{UW}} \mathbf{x}_{\mathrm{UW}}^{\mathrm{H}} \tilde{\mathbf{H}}_{\mathrm{UW}_{u^{\prime}}}^{\mathrm{H}}\right\} \mathbf{U D}^{\mathrm{H}} \mathbf{F}_{K}^{\mathrm{H}}+\mathbf{F}_{K} \mathbf{D} \mathbf{D}^{\mathrm{H}} \mathbf{F}_{K}^{\mathrm{H}} \sigma^{2} \delta\left[u-u^{\prime}\right] \\
& \stackrel{(c)}{=} \mathbf{F}_{K} \mathbf{D} \mathbf{U}^{\mathrm{H}} \mathbf{R}\left(u, u^{\prime}\right) \mathbf{U} \mathbf{D}^{\mathrm{H}} \mathbf{F}_{K}^{\mathrm{H}}+\mathbf{F}_{K} \mathbf{D D}^{\mathrm{H}} \mathbf{F}_{K}^{\mathrm{H}} \sigma^{2} \delta\left[u-u^{\prime}\right] \\
& \mathbb{E}\left\{\hat{\mathbf{P}}_{k} \hat{\mathbf{P}}_{k}^{\mathrm{H}}\right\}=\left[\begin{array}{cc}
\left(\mathbf{F}_{K} \mathbf{D} \mathbf{U}^{\mathrm{H}} \mathbf{R}(0,0) \mathbf{U} \mathbf{D}^{\mathrm{H}} \mathbf{F}_{K}^{\mathrm{H}}\right)_{k, k}+\frac{L}{N} \sigma^{2} & \left(\mathbf{F}_{K} \mathbf{D} \mathbf{U}^{\mathrm{H}} \mathbf{R}(0,1) \mathbf{U D}^{\mathrm{H}} \mathbf{F}_{K}^{\mathrm{H}}\right)_{k, k} \\
\left(\mathbf{F}_{K} \mathbf{D} \mathbf{D} \mathbf{U}^{\mathrm{H}} \mathbf{R}(1,0) \mathbf{U} \mathbf{D}^{\mathrm{H}} \mathbf{F}_{K}^{\mathrm{H}}\right)_{k, k} & \left(\mathbf{F}_{K} \mathbf{D} \mathbf{U}^{\mathrm{H}} \mathbf{R}(1,1) \mathbf{U} \mathbf{D}^{\mathrm{H}} \mathbf{F}_{K}^{\mathrm{H}}\right)_{k, k}+\frac{L}{N} \sigma^{2}
\end{array}\right]
\end{aligned}
$$


averaged time-varying impulse response of $\mathbf{H}_{m}$ as in Equation (20). Secondly, we approach this derivation by using a similar mathematical procedure as (44), such have a solution in an analogous format for simplicity. Therefore, we the averaged channel response in the frequency domain related to the $m$-th subblock as

$$
\operatorname{diag}\left(\boldsymbol{\Lambda}_{m}\right)=\left(\mathbf{F}_{K} \overline{\mathbf{h}}_{m}\right)=\left(\mathbf{F}_{K} \mathbf{D} \mathbf{U}^{\mathrm{H}} \overline{\mathbf{H}}_{m}^{\prime} \mathbf{x}_{\mathrm{UW}}\right),
$$

where $\overline{\mathbf{h}}_{m}=\left(\overline{\mathbf{H}}_{m}\right)_{*, 0}$ is the averaged channel impulse response, and the matrix $\overline{\mathbf{H}}_{m}^{\prime} \in \mathbb{C}^{N_{\mathrm{UW}} \times N_{\mathrm{UW}}}$ is a circulant matrix whose first column is $\left(\overline{\mathbf{H}}_{m}^{\prime}\right)_{*, 0}=\left[\overline{\mathbf{h}}_{m}\right]_{n=0}^{N_{\mathrm{UW}}-1}$. Also, one can observe that $\overline{\mathbf{h}}_{m}=\mathbf{D} \mathbf{U}^{\mathrm{H}} \overline{\mathbf{H}}_{m} \mathbf{x}_{\mathrm{UW}}$ by considering that the LS estimating matrix $\mathbf{U}^{\mathrm{H}}$ returns the exact channel in the absence of noise. And the matrix $\mathbf{D}$ guarantee an $K$ length vector. We emphasize that writing $\operatorname{diag}\left(\boldsymbol{\Lambda}_{m}\right)$ depending on the UW in (45) is an strategy to provide a closed form solution in an analogous manner to (44), such that we can reuse some of the concepts considered in the previous subsection.

The correlation between the estimated channel of the $u$-th UW with the averaged channel given in the rightmost part of (45) is given in the equation (46). The line (b) of (46) is obtained analogously to the derivation of equation (41). The only term related to the AWGN that remains is its first moment, which is zero. Thus, there is no AWGN term in (46). Moreover, equation (46) $(c)$ has the matrix $\mathbf{S}(m, u) \in \mathbb{C}^{N_{\mathrm{UW}} \times N_{\mathrm{UW}}}$ which analogously to (42) is obtained as

$$
\begin{aligned}
& (\mathbf{S}(m, u))_{n, n^{\prime}} \\
& =\operatorname{Tr}\left\{\left(\mathbf{x}_{\mathrm{UW}} \mathbf{x}_{\mathrm{UW}}^{\mathrm{H}}\right) \operatorname{shift}_{n^{\prime}, n}\left\{\overline{\boldsymbol{\Sigma}}_{\mathbf{H}_{\mathrm{UW}}}\left(n^{\prime}, m, u\right)\right\}\right\},
\end{aligned}
$$

where

$$
\begin{aligned}
& \overline{\boldsymbol{\Sigma}}_{\mathbf{H}_{\mathrm{UW}}}\left(n^{\prime}, m, u\right) \\
& =\left[\begin{array}{cccc}
\rho_{0} & & & \\
& 0 & & \\
& & \ddots & \\
& & & \rho_{1}
\end{array}\right] \frac{1}{N} \sum_{i=0}^{N-1} \Upsilon\left(n^{\prime}+u \Delta_{u}-\left(N_{\mathrm{ini}_{m}}+i\right)\right) .
\end{aligned}
$$

The correlation coefficient in (48) considers the linear combination of all channel values present in the averaged channel $\overline{\mathbf{h}}_{m}$, with the channel response at the $n^{\prime}$-th time index related to the $u$-th UW. $N_{\text {ini }_{m}}=N_{\mathrm{CP}_{\mathrm{uw}}}+N_{\mathrm{UW}}+N_{\mathrm{CP}}+m\left(N_{\mathrm{CP}}+K\right)$ is the starting channel sample for the $m$-th subblock. Thus, we combine (47) with (46) and finally obtained the closed form solution

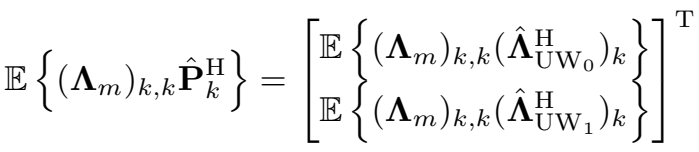

$$
\begin{aligned}
& =\left[\begin{array}{l}
\left(\mathbf{F}_{K} \mathbf{D} \mathbf{U}^{\mathrm{H}} \mathbf{S}(m, 0) \mathbf{U} \mathbf{D}^{\mathrm{H}} \mathbf{F}_{K}^{\mathrm{H}}\right)_{k, k} \\
\left(\mathbf{F}_{K} \mathbf{D} \mathbf{U}^{\mathrm{H}} \mathbf{S}(m, 1) \mathbf{U} \mathbf{D}^{\mathrm{H}} \mathbf{F}_{K}^{\mathrm{H}}\right)_{k, k}
\end{array}\right]^{\mathrm{T}},
\end{aligned}
$$

where we are interested in the diagonal terms of (46), thus we take the elements $(\cdot)_{k, k}$.

\section{APPENDIX B \\ POWER OF AVERAGED CHANNEL}

In this appendix, we show that $\mathbb{E}\left\{\left|\left(\boldsymbol{\Lambda}_{m}\right)_{k, k}\right|^{2}\right\}=$ $1 / K^{2} \sum_{i=0}^{K-1} \sum_{j=0}^{K-1} \Upsilon(i-j) \forall_{k, m}$. Firstly, we notice that $\left(\boldsymbol{\Lambda}_{m}\right)_{k, k}=\left(\mathbf{F} \mathbf{H}_{m} \mathbf{F}^{\mathrm{H}}\right)_{k, k}$ as stated by Equation (19). Thus, we can write

$$
\mathbb{E}\left\{\left|\left(\boldsymbol{\Lambda}_{m}\right)_{k, k}\right|^{2}\right\}=\left(\mathbf{F E}\left\{\overline{\mathbf{H}}_{m} \overline{\mathbf{H}}_{m}^{\mathrm{H}}\right\} \mathbf{F}^{\mathrm{H}}\right)_{k, k}
$$

where the Fourier matrix are left outside the expectation operator because it is a constant. Combining the last equation with (20), we can solve the expectation as

$$
\begin{aligned}
& \left(\mathbb{E}\left\{\overline{\mathbf{H}}_{m} \overline{\mathbf{H}}_{m}^{\mathrm{H}}\right\}\right)_{n, n^{\prime}} \\
& =\frac{1}{K^{2}} \sum_{i=0}^{K-1} \sum_{j=0}^{K-1} \mathbb{E}\left\{\left(\mathbf{H}_{m}\right)_{(i+n)_{K}, i}\left(\mathbf{H}_{m}\right)_{\left(j+n^{\prime}\right)_{K}, j}^{\mathrm{H}}\right\} \\
& =\frac{1}{K^{2}} \sum_{i=0}^{K-1} \sum_{j=0}^{K-1} \Upsilon(i-j)
\end{aligned}
$$

for $n=n^{\prime}$ and zero otherwise due to no correlation among different taps in the channel's impulse response (3). The mid equation of (51) rewrites $\mathbb{E}\left\{\overline{\mathbf{H}}_{m} \overline{\mathbf{H}}_{m}^{\mathrm{H}}\right\}$ with all cross terms of the averaged impulse response. The temporal difference among these cross terms results in the correlation factor of the rightmost equality of (51). Then, we see that $\mathbb{E}\left\{\overline{\mathbf{H}}_{m} \overline{\mathbf{H}}_{m}^{\mathrm{H}}\right\}$ is a diagonal matrix with equal elements because they are independent of $n$. Therefore the Fourier matrices in (50) will result in the identity matrix and can be neglected. Finally, (51) does not depend on the index $m$, concluding this proof.

\section{APPENDIX C \\ DOPPLER ERROR}

Generally, by combining Equation (21) with (19), we can define the Doppler $\sigma_{\mathrm{D}_{m, k}}^{2}=\mathbb{E}\left\{\left(\boldsymbol{\Lambda}_{\mathrm{DE}_{m}} \boldsymbol{\Lambda}_{\mathrm{DE}_{m}}^{\mathrm{H}}\right)_{k, k}\right\}$ error

$$
\begin{aligned}
\mathbb{E}\left\{\operatorname{diag}\left(\boldsymbol{\Lambda}_{m}\right) \hat{\mathbf{\Lambda}}_{\mathrm{UW}_{u}}^{\mathrm{H}}\right\} & \stackrel{(a)}{=} \mathbb{E}\left\{\mathbf{F}_{K} \mathbf{D} \mathbf{U}^{\mathrm{H}} \overline{\mathbf{H}}_{m}^{\prime} \mathbf{x}_{\mathrm{UW}}\left(\tilde{\mathbf{H}}_{\mathrm{UW}_{u}} \mathbf{x}_{\mathrm{UW}}+\mathbf{w}_{\mathrm{UW}_{u}}\right)^{\mathrm{H}} \mathbf{U D}^{\mathrm{H}} \mathbf{F}_{K}^{\mathrm{H}}\right\} \\
& \stackrel{(b)}{=} \mathbf{F}_{K} \mathbf{D} \mathbf{U}^{\mathrm{H}} \mathbb{E}\left\{\overline{\mathbf{H}}_{m}^{\prime} \mathbf{x}_{\mathrm{UW}} \mathbf{x}_{\mathrm{UW}}^{\mathrm{H}} \tilde{\mathbf{H}}_{\mathrm{UW}}^{\mathrm{H}}\right\} \mathbf{U D}^{\mathrm{H}} \mathbf{F}_{K}^{\mathrm{H}} \\
& \stackrel{(c)}{=} \mathbf{F}_{K} \mathbf{D} \mathbf{U}^{\mathrm{H}} \mathbf{S}(m, u) \mathbf{U} \mathbf{D}^{\mathrm{H}} \mathbf{F}_{K}^{\mathrm{H}}
\end{aligned}
$$


power as

$$
\begin{aligned}
& \sigma_{\mathrm{D}_{m, k}}^{2} \\
& =\left(\mathbb{E}\left\{\mathbf{F}\left(\mathbf{H}_{m}-\overline{\mathbf{H}}_{m}\right) \mathbf{F}^{\mathrm{H}}\right\}\right)_{k, k} \\
& =\left(\mathbf{F}\left(\mathbb{E}\left\{\mathbf{H}_{m} \mathbf{H}_{m}^{\mathrm{H}}\right\}-2 \mathbb{E}\left\{\mathbf{H}_{m} \overline{\mathbf{H}}_{m}^{\mathrm{H}}\right\}+\mathbb{E}\left\{\overline{\mathbf{H}}_{m} \overline{\mathbf{H}}_{m}^{\mathrm{H}}\right\}\right) \mathbf{F}^{\mathrm{H}}\right)_{k, k}
\end{aligned}
$$

where $\mathbb{E}\left\{\mathbf{H}_{m} \mathbf{H}_{m}^{\mathrm{H}}\right\}=\mathbf{I}$ due to the assumption of $\sum_{l=0}^{L-1} \rho_{l}=$ 1 in (3) and no intertap correlation. Moreover, $\mathbb{E}\left\{\mathbf{H}_{m} \overline{\mathbf{H}}_{m}^{\mathrm{H}}\right\}$ has been already computed in Equation (51). The remaining expectation $\mathbb{E}\left\{\mathbf{H}_{m} \overline{\mathbf{H}}_{m}^{\mathrm{H}}\right\}$ is found analogously to (51) and is given by

$$
\left(\mathbb{E}\left\{\mathbf{H}_{m} \overline{\mathbf{H}}_{m}^{\mathrm{H}}\right\}\right)_{n, n^{\prime}}=\frac{1}{K} \sum_{i=0}^{K-1} \Upsilon(i-n)
$$

for $n=n^{\prime}$ and zero otherwise due the assumption of no intertap correlation. Since the last expectation defines a diagonal matrix, it follows that

$$
\begin{aligned}
\operatorname{diag}\left(\mathbf{F E}\left\{\mathbf{H}_{m} \overline{\mathbf{H}}_{m}^{\mathrm{H}}\right\} \mathbf{F}^{\mathrm{H}}\right) & =\frac{\mathbf{1}_{K}}{K} \operatorname{Tr}\left(\mathbb{E}\left\{\mathbf{H}_{m} \overline{\mathbf{H}}_{m}^{\mathrm{H}}\right\}\right) \\
& =\frac{\mathbf{1}_{K}}{K} \sum_{n=0}^{K-1} \frac{1}{K} \sum_{i=0}^{K-1} \Upsilon(i-n)
\end{aligned}
$$

whose mid equation is straightforward to check due to the construction of $\mathbf{F}$, and whose rightmost side is identical to the Equation (51). Thus, combining the last equation with (52) leads to Equation (23).

\section{REFERENCES}

[1] 3GPP Technical Specification (TS) 38.300, "NR; NR and NG-RAN Overall Description; Stage 2, Rel. 15," tech. rep., v15.2.0, 2018.

[2] "LTE; Evolved Universal Terrestrial Radio Access (E-UTRA) and Evolved Universal Terrestrial Radio Access Network (E-UTRAN); Overall description, 3GPP Standard TS 36.300, 2011.,"

[3] Y. Carmon, S. Shamai, and T. Weissman, "Comparison of the Achievable Rates in OFDM and Single Carrier Modulation with I.I.D. Inputs," IEEE Trans. Inf. Theory, vol. 61, pp. 1795-1818, April 2015.

[4] R. Bomfin, D. Zhang, M. Matthé, and G. Fettweis, "A Theoretical Framework for Optimizing Multicarrier Systems Under Time and/or Frequency-Selective Channels," IEEE Commun. Lett., vol. 22, pp. 2394 2397, Nov 2018.

[5] R. Bomfin, M. Chafii, and G. Fettweis, "Low-Complexity Iterative Receiver for Orthogonal Chirp Division Multiplexing," in IEEE WCNC workshops, (Marrakech, Morocco), Apr. 2019.

[6] R. Hadani, S. Rakib, M. Tsatsanis, A. Monk, A. J. Goldsmith, A. F. Molisch, and R. Calderbank, "Orthogonal Time Frequency Space Modulation," in 2017 IEEE Wireless Communications and Networking Conference (WCNC), pp. 1-6, March 2017.

[7] A. Nimr, M. Chafii, M. Matthe, and G. Fettweis, "Extended GFDM framework: OTFS and GFDM comparison," in 2018 IEEE global communications conference (GLOBECOM), pp. 1-6, IEEE, 2018.

[8] T. Zemen, M. Hofer, D. Löschenbrand, and C. Pacher, "Iterative Detection for Orthogonal Precoding in Doubly Selective Channels," in 2018 IEEE 29th Annual International Symposium on Personal, Indoor and Mobile Radio Communications (PIMRC), pp. 1-7, 2018.

[9] T. Zemen, D. Löschenbrand, M. Hofer, C. Pacher, and B. Rainer, "Orthogonally precoded massive mimo for high mobility scenarios," IEEE Access, vol. 7, pp. 132979-132990, 2019.

[10] R. Bomfin, A. Nimr, M. Chafii, and G. Fettweis, "A Robust and Low-Complexity Walsh-Hadamard Modulation for Doubly-Dispersive Channels," IEEE Commun. Lett., pp. 1-1, 2020.

[11] L. Deneire, B. Gyselinckx, and M. Engels, "Training sequence versus cyclic prefix-a new look on single carrier communication," IEEE COmmun. Lett., vol. 5, no. 7, pp. 292-294, 2001.
[12] S. Ehsanfar, M. Chafii, and G. Fettweis, "On UW-based Transmission for MIMO Multi-Carriers with Spatial Multiplexing," IEEE Trans. Wireless Commun., pp. 1-1, 2020.

[13] Y. R. Zheng and C. Xiao, "Channel Estimation for Frequency-Domain Equalization of Single-Carrier Broadband Wireless Communications," IEEE Trans. Veh. Technol., vol. 58, no. 2, pp. 815-823, 2009.

[14] R. Bomfin, M. Chafii, and G. Fettweis, "Novel Iterative Receiver Design for CP-Free Transmission Under Frequency-Selective Channels," IEEE Commun. Lett., vol. 24, no. 3, pp. 525-529, 2020.

[15] X. Liu, H. Chen, S. Chen, and W. Meng, "Symbol Cyclic-Shift Equalization Algorithm - A CP-Free OFDM/OFDMA System Design," IEEE Trans. Veh. Technol., vol. 66, pp. 282-294, Jan 2017.

[16] J. Coon, M. Sandell, M. Beach, and J. McGeehan, "Channel and noise variance estimation and tracking algorithms for unique-word based single-carrier systems," IEEE Trans. Commun., vol. 5, no. 6, pp. 14881496, 2006.

[17] S. Ehsanfar, M. Chafii, and G. Fettweis, "A Frame Design for MIMO UW based Systems: Overhead Analysis Channel Estimation," in 2019 IEEE 2nd 5G World Forum (5GWF), pp. 173-178, 2019.

[18] R. H. Clarke, "A statistical theory of mobile-radio reception," The Bell System Technical Journal, vol. 47, no. 6, pp. 957-1000, 1968.

[19] X. Ouyang and J. Zhao, "Orthogonal Chirp Division Multiplexing," IEEE Trans. Commun., vol. 64, pp. 3946-3957, Sept 2016.

[20] C. Studer, S. Fateh, and D. Seethaler, "ASIC Implementation of SoftInput Soft-Output MIMO Detection Using MMSE Parallel Interference Cancellation," IEEE J. Solid-State Circuits, vol. 46, pp. 1754-1765, July 2011.

[21] M. Matthé, D. Zhang, and G. Fettweis, "Low-complexity iterative mmsepic detection for mimo-gfdm," IEEE Trans. Commun., vol. 66, pp. 14671480, April 2018.

[22] G. A. F. Seber and A. J. Lee, Linear Regression Analysis, 2nd Edition. Wiley, 2003.

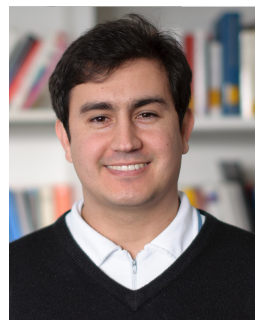

Roberto Bomfin received his B.Sc. and M.Sc degrees in Electrical Engineering from the Instituto Nacional de Telecomunicações (INATEL), Brazil, in 2014 and 2016, respectively. From 2013 to 2015, he worked as teaching assistant in the undergraduate study programm of of INATEL. In 2014, he worked as a researcher for the brazilian undergraduate research program in spectrum sensing for cognitive radio networks. In September of 2016, he joined the Vodafone Chair Mobile Communications Systems at the Technische Universität Dresden (TUD) in Dresden, Germany, as Ph.D. student. His research and interest topics include waveform and receiver design, non-orhogonal multiple access (NOMA), and joint communication and sensing. 


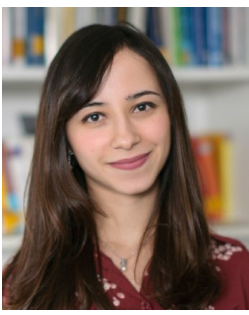

Marwa Chafii received her Ph.D. degree in Telecommunications in 2016, and her Master's degree in the field of advanced wireless communication systems (SAR) in 2013, both from CentraleSupélec, France. Between 2014 and 2016, she has been a visiting researcher at Poznan University of Technology (Poland), University of York (UK), Yokohama National University (Japan), and University of Oxford (UK). She joined the Vodafone Chair Mobile Communication Systems at the Technical University of Dresden, Germany, in February 2018 as a research group leader. Since September 2018, she is research projects lead at Women in AI and an associate professor at ENSEA, France, where she holds a Chair of Excellence on Artificial Intelligence from CY Initiative. She received the prize of the best Ph.D. in France in the field of Signal, Image and Vision, and she has been nominated in the top 10 Rising Stars in Computer Networking and Communications by N2Women in 2020. Her research interests include machine learning for wireless communications, advanced waveform design, indoor localization and channel estimation. Since 2019, she serves as Associate Editor at IEEE Communications Letters where she received the Best Editor Award in 2020. She is currently vice-chair of the IEEE ComSoc Emerging Technology Initiative on Machine Learning for Communications and managing the Gender Committee of the AI4EU community.

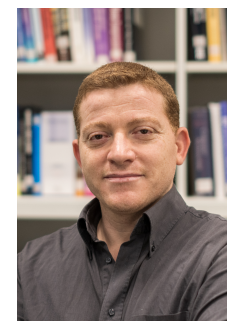

Ahmad Nimr received the Diploma degree in communication engineering, in 2004, and the M.Sc. degree in communications and signal processing from TU Ilmenau, in 2014. He worked as a Software and Hardware Developer from 2005 to 2011 . He has been a member of the Vodafone Chair at TU Dresden since October 2015. His research activities focus on multicarrier waveforms and multiple access techniques. He is also involved in the design and implementation of real-time communication systems. his excellent master's grades.

He received the Best Graduate Student Award for

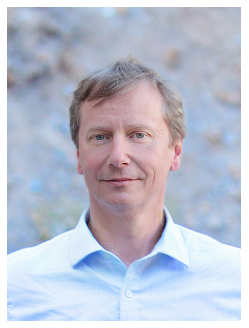

Gerhard Fettweis is Vodafone Chair Professor at TU Dresden since 1994, and heads the Barkhausen Institute since 2018, respectively. He earned his Ph.D. under H. Meyr's supervision from RWTH Aachen in 1990. After one year at IBM Research in San Jose, CA, he moved to TCSI Inc., Berkeley, CA. He coordinates the 5G Lab Germany, and 2 German Science Foundation (DFG) centers at TU Dresden, namely cfaed and HAEC. His research focusses on wireless transmission and chip design for wireless/IoT platforms, with 20 companies from Asia/Europe/US sponsoring his research. Gerhard is IEEE Fellow, member of the German Academy of Sciences (Leopoldina), the German Academy of Engineering (acatech), and received multiple IEEE recognitions as well has the VDE ring of honor. In Dresden his team has spun-out sixteen start-ups, and setup funded projects in volume of close to EUR 1/2 billion. He co-chairs the IEEE 5G Initiative, and has helped organizing IEEE conferences, most notably as TPC Chair of ICC 2009 and of TTM 2012, and as General Chair of VTC Spring 2013 and DATE 2014. 